J. DIFFERENTIAL GEOMETRY

60 (2002) 485-521

\title{
SELFDUAL EINSTEIN METRICS WITH TORUS SYMMETRY
}

\author{
DAVID M.J. CALDERBANK \& HENRIK PEDERSEN
}

\begin{abstract}
It is well-known that any 4-dimensional hyperkähler metric with two commuting Killing fields may be obtained explicitly, via the Gibbons-Hawking Ansatz, from a harmonic function invariant under a Killing field on $\mathbb{R}^{3}$. In this paper, we find all selfdual Einstein metrics of nonzero scalar curvature with two commuting Killing fields. They are given explicitly in terms of a local eigenfunction of the Laplacian on the hyperbolic plane. We discuss the relation of this construction to a class of selfdual spaces found by Joyce, and some Einstein-Weyl spaces found by Ward, and then show that certain 'multipole' hyperbolic eigenfunctions yield explicit formulae for the quaternion-kähler quotients of $\mathbb{H} P^{m-1}$ by an $(m-2)$-torus studied by Galicki and Lawson. As a consequence we are able to place the well-known cohomogeneity one metrics, the quaternion-kähler quotients of $\mathbb{H} P^{2}$ (and noncompact analogues), and the more recently studied selfdual Einstein Hermitian metrics in a unified framework, and give new complete examples.
\end{abstract}

\section{Introduction}

We present in this paper an explicit classification of 4-dimensional Einstein metrics with selfdual Weyl curvature and two linearly independent commuting Killing fields. We refer to these metrics as selfdual Einstein metrics with torus symmetry, since they are the local form (on a dense open set) of such metrics with an action of $T^{2}, S^{1} \times \mathbb{R}$ or $\mathbb{R}^{2}$ by isometries.

When the selfdual Einstein metric $g$ is scalar-flat, it is well-known that $g$ is locally hyperkähler and that some linear combination of the two Killing fields is triholomorphic - hence $g$ is determined by a harmonic function on $\mathbb{R}^{3}$, via the Gibbons-Hawking Ansatz [21], and this harmonic

Received May 23, 2002. 
function is invariant under the transrotation of $\mathbb{R}^{3}$ coming from the second Killing field of $g$. Therefore we focus on the case that the selfdual Einstein metric has nonzero scalar curvature.

Theorem 1.1. Let $F(\rho, \eta)$ be a solution of the linear differential equation

$$
F_{\rho \rho}+F_{\eta \eta}=\frac{3 F}{4 \rho^{2}}
$$

on some open subset of the half-space $\rho>0$, and consider the metric $g(\rho, \eta, \phi, \psi)$ given by

$$
\begin{aligned}
g= & \frac{F^{2}-4 \rho^{2}\left(F_{\rho}^{2}+F_{\eta}^{2}\right)}{4 F^{2}} \frac{d \rho^{2}+d \eta^{2}}{\rho^{2}} \\
& +\frac{\left(\left(F-2 \rho F_{\rho}\right) \alpha-2 \rho F_{\eta} \beta\right)^{2}+\left(-2 \rho F_{\eta} \alpha+\left(F+2 \rho F_{\rho}\right) \beta\right)^{2}}{F^{2}\left(F^{2}-4 \rho^{2}\left(F_{\rho}^{2}+F_{\eta}^{2}\right)\right)}
\end{aligned}
$$

where $\alpha=\sqrt{\rho} d \phi$ and $\beta=(d \psi+\eta d \phi) / \sqrt{\rho}$. Then:

(i) On the open set where $F^{2}>4 \rho^{2}\left(F_{\rho}^{2}+F_{\eta}^{2}\right), g$ is a selfdual Einstein metric of positive scalar curvature, whereas on the open set where $0<F^{2}<4 \rho^{2}\left(F_{\rho}^{2}+F_{\eta}^{2}\right),-g$ is a selfdual Einstein metric of negative scalar curvature.

(ii) Any selfdual Einstein metric of nonzero scalar curvature with two linearly independent commuting Killing fields arises locally in this way, (i.e., in a neighbourhood of any point, it is of the form (1.1) up to a constant multiple).

The metric $g$ is sufficiently explicit to make it straightforward, though tedious, to check that it is selfdual and Einstein, for instance, by computing the curvature of the Levi-Civita connection of $g$ on antiselfdual 2-forms using the Cartan calculus [37]. Hence the heart of the above theorem is part (ii), so we concentrate on this and explain why all selfdual Einstein metrics with torus symmetry are of this form. The proof of (ii) will in fact encode the selfdual Einstein condition (using work of Tod [39]), thus proving (i) at the same time.

There are three features of these metrics which need to be explained. First, and most remarkable, is the fact that the equation for $F$ is linear. This means that we can "superpose" two such metrics to yield a third.

Second, the equation for $F$ means that it is a local eigenfunction, with eigenvalue $3 / 4$, of the Laplacian $\rho^{-2}\left(\partial_{\rho}^{2}+\partial_{\eta}^{2}\right)$ of the hyperbolic 
metric $\left(d \rho^{2}+d \eta^{2}\right) / \rho^{2}$. In other words $F$ satisfies a natural differential equation on the hyperbolic plane.

Third, the level surfaces of constant $\phi, \psi$ are orthogonal to both Killing fields $\partial / \partial \phi$ and $\partial / \partial \psi$, hence the orbits of the induced 2-dimensional local symmetry group are surface-orthogonal, i.e., the orthogonal distribution to the orbits is integrable.

These three features are closely related. We originally found an explicit form for selfdual Einstein metrics with torus symmetry by noticing that the local quotient of such a metric by one of its Killing fields must be an Einstein-Weyl space with an axial symmetry, i.e., one of the spaces found by Ward [40] and studied in [9]. These Einstein-Weyl spaces are given explicitly in terms of an axisymmetric harmonic function (AHF) on $\mathbb{R}^{3}$, i.e., a solution of a linear differential equation. The geometry of the hyperbolic plane $\mathcal{H}^{2}$ enters the picture because $\mathbb{R}^{3} \backslash \mathbb{R}$ is conformal to $\mathcal{H}^{2} \times S^{1}$. Finally, these Einstein-Weyl spaces are the quotients of the conformal metrics found by Joyce [26], who obtained a classification of selfdual 4-manifolds with two commuting surface-orthogonal conformal vector fields.

This original argument had the advantage of being an exercise in pure thought, i.e., a combination of known results with no new computations. However, it led to a rather awkward description of the conformally Einstein metrics among Joyce's selfdual spaces.

Therefore, we shall present a different, more self-contained proof of Theorem 1.1. Indeed, we proceed rather in reverse, by establishing directly, in Section 3, the surface-orthogonality of the Killing fields. To do this, we first review, in Section 2, an isomorphism between Killing fields and twistors which plays an important role throughout the paper - these twistors are essentially the same thing as compatible scalar-flat Kähler metrics [33]. We discuss this in Section 4, together with Tod's description [39] of selfdual Einstein metrics with a Killing field in terms of the $\mathrm{SU}(\infty)$ Toda equation (see also $[34,15]$ ).

Returning to the general argument, the surface-orthogonality of the Killing fields shows that the conformal structure of a selfdual Einstein metric with torus symmetry is a 'Joyce space' [26]. We review the theory of Joyce spaces and their quotient Einstein-Weyl spaces in Section 5 and explain how they are determined by two solutions of a spinor equation on $\mathcal{H}^{2}$ which is equivalent to the equation for AHFs. On the other hand, Tod's analysis of selfdual Einstein metrics with a Killing field shows that these two AHFs must be related in a special way. The essential idea is that the AHFs are constructed from the eigenfunction $F$ by a Bäcklund 
transformation, and a 2-dimensional family is obtained because this transformation is coordinate dependent. In Sections 6 and 7 we use the spin geometry of the hyperbolic plane to give a more natural description, and this allows us to complete the proof of Theorem 1.1.

In Section 8 we discuss the Swann bundle [36] of our selfdual Einstein metrics, cf. [13]. This hyperkähler 8-manifold with two commuting triholomorphic Killing fields is given locally by the generalized GibbonsHawking construction of $[23,32]$ and we establish the relationship between this construction and the eigenfunction $F$. Then, comparing the Swann bundle with the hyperkähler quotients of $\mathbb{H}^{m}$ by an $(m-2)$ torus (see [5]), we are led, in Section 9, to define 'multipole' hyperbolic eigenfunctions and prove the following theorem:

Theorem 1.2. The selfdual Einstein metrics arising as quaternionkähler quotients of quaternionic projective space $\mathbb{H} P^{m-1}$ by an $(m-2)$ dimensional family of commuting Killing fields are exactly the metrics given by (1.1) where $F$ is a 'positive m-pole solution' of the hyperbolic eigenfunction equation on $\mathcal{H}^{2}$.

A more precise statement is given in Theorem 9.1. In particular, this characterizes the $m$-pole solutions corresponding to quaternion-kähler quotients of $\mathbb{H} P^{m-1}$ by an $(m-2)$-torus, which yield the compact selfdual Einstein orbifold metrics of Galicki-Lawson [19] when $m=3$, and Boyer-Galicki-Mann-Rees [7] in general.

Replacing $\mathbb{H}^{m}$ with $\mathbb{H}^{p, q}$ leads to noncompact analogues of these metrics, among which there are examples of complete selfdual Einstein metrics of negative scalar curvature. Some of these metrics are wellknown - and our approach provides a unified description of them but we also obtain new examples.

Acknowledgements. The first author would like to thank Vestislav Apostolov, Roger Bielawski, Paul Gauduchon, Kris Galicki, Elmer Rees, and Michael Singer for stimulating discussions, and also the EPSRC, the Leverhulme Trust and the William Gordon Seggie Brown Trust for the Research Fellowships that enabled him to carry out this research. Both authors are members of EDGE, Research Training Network HPRN-CT2000-00101, supported by the European Human Potential Programme 


\section{Selfdual Einstein metrics, twistors and Killing fields}

We begin by reviewing the relation between Killing fields and antiselfdual twistors on a selfdual Einstein manifold, following [4, 39]. Recall that a vector field $K$ is a Killing field of a metric $g$ if and only if its covariant derivative $D^{g} K$ is a skew endomorphism of the tangent bundle (the endomorphism is defined by $X \mapsto D_{X}^{g} K$ ).

In four dimensions skew endomorphisms decompose into selfdual and antiselfdual parts: $\mathfrak{s o}(T M)=\mathfrak{s o}_{+}(T M) \oplus \mathfrak{s o}_{-}(T M)$. This is related (via the metric) to the decomposition $\wedge^{2} T^{*} M=\wedge_{+}^{2} T^{*} M \oplus \wedge_{-}^{2} T^{*} M$ of the bundle of 2-forms into eigenspaces of the Hodge $*$ operator. We denote the decomposition of sections of these bundles by $A=A^{+}+A^{-}$.

An antiselfdual endomorphism $\Psi$ is called a twistor if there is a 1form $\gamma$ such that $D_{X}^{g} \Psi=(\gamma \triangle X)^{-}$for all vector fields $X$ - here, for vector fields $X, Y$, we define $\gamma \triangle X(Y)=\gamma(Y) X-\langle X, Y\rangle \sharp \gamma$. It follows by taking a trace that $\gamma$ is a multiple of the divergence $\delta^{g} \Psi$ and so this is a linear differential equation on $\Psi$, called the twistor equation.

For a selfdual Einstein metric, the curvature $R^{g} \in \mathrm{C}^{\infty}\left(M, \wedge^{2} T^{*} M \otimes\right.$ $\mathfrak{s o}(T M)$ ) has only two irreducible components: the selfdual Weyl curvature $W^{+} \in \mathrm{C}^{\infty}\left(M, \wedge_{+}^{2} T^{*} M \otimes \mathfrak{s o}_{+}(T M)\right)$, and the (normalized) scalar curvature $s^{g}=\frac{1}{6} \mathrm{scal}^{g}$.

This has strong consequences for Killing fields and twistors.

Proposition 2.1. Let $g$ be a selfdual Einstein metric.

(i) Suppose $K$ is a Killing field of $g$ and let $\Psi=\left(D^{g} K\right)^{-}$. Then $D_{X}^{g} \Psi=\frac{1}{2} s^{g}(\langle K, \cdot\rangle \triangle X)^{-}$and so $\Psi$ is a twistor.

(ii) Suppose $\Psi$ is a twistor with $D_{X}^{g} \Psi=(\gamma \Delta X)^{-}$. Then the dual vector field $\sharp^{g} \gamma$ is a Killing field of $g$ and $\left(D^{g} \gamma\right)^{-}=\frac{1}{2} s^{g}\langle\Psi(\cdot), \cdot\rangle$.

Proof. (i) Since $K$ is Killing and $g$ is selfdual Einstein, we have

$$
D_{X}^{g} D^{g} K=R_{X, K}^{g}=W_{X, K}^{+}+\frac{1}{2} s^{g}\langle K, \cdot\rangle \triangle X .
$$

The antiselfdual part of this is what we want.

(ii) Differentiating the twistor equation again and skew symmetrizing gives

$$
\left(D_{X}^{g} \gamma \Delta Y\right)^{-}-\left(D_{Y}^{g} \gamma \Delta X\right)^{-}=\left[R_{X, Y}^{g}, \Psi\right]=-\frac{1}{2} s^{g}[\langle X, \cdot\rangle \Delta Y, \Psi] .
$$


Contracting with another vector field $Z$ and taking the trace over $X$ and $Z$, we obtain

$$
\operatorname{sym} D^{g} \gamma+\frac{1}{2} \delta^{g} \gamma+(d \gamma)^{-}=s^{g}\langle\Psi(\cdot), \cdot\rangle \text {. }
$$

The right-hand side is skew, so $D^{g} \gamma$ is skew, $\sharp^{g} \gamma$ is a Killing field and $2\left(D^{g} \gamma\right)^{-}=s^{g}\langle\Psi(\cdot), \cdot\rangle$.

q.e.d.

Corollary 2.2. Let $g$ be a selfdual Einstein metric of nonzero scalar curvature. Then there is a linear isomorphism from space of Killing fields of $g$ to the space of twistors.

This is not true if $g$ has zero scalar curvature, when $D^{g}$ is flat on $\mathfrak{s o}_{-}(T M)$ and $g$ is locally hyperkähler. Then Proposition 2.1 (i) says that $\left(D^{g} K\right)^{-}$is parallel, and so $K$ is either triholomorphic, or $\left(D^{g} K\right)^{-}$ is a nonzero constant multiple of one of the complex structures. Proposition 2.1 (ii) says that the Killing field associated to a twistor is triholomorphic.

Let us remark, however, that this isomorphism does generalize to quaternion-kähler manifolds of nonzero scalar curvature [35], and underlies the quaternion-kähler quotient [19]. 4m-dimensional quaternionkähler manifolds with $T^{m+1}$-symmetry have beed studied in [13].

\section{Surface-orthogonality}

In this section we show that the orbits of two commuting Killing fields of a selfdual Einstein metric with nonzero scalar curvature are necessarily surface-orthogonal.

Recall that a Killing vector $K$ is hypersurface-orthogonal if and only if $\left(D^{g} K\right)(X, Y)=0$ for all $X, Y$ orthogonal to $K$. Here we view $D^{g} K$ as a 2-form using the metric $g ;\left(D^{g} K\right)(X, Y)=-\frac{1}{2}\langle K,[X, Y]\rangle$ and so hypersurface-orthogonality means precisely that the orthogonal distribution is integrable. Equivalently $K$ is hypersurface-orthogonal if and only if $\left(* D^{g} K\right)(K)$ is zero. In four dimensions, this is a 1-form called the twist of $K$.

Similarly, two linearly independent Killing vector fields $K, \widetilde{K}$ are (codimension 2-) surface-orthogonal if and only if $\left(D^{g} K\right)(X, Y)=0$ and $\left(D^{g} \widetilde{K}\right)(X, Y)=0$ for all $X, Y$ orthogonal to both $K$ and $\widetilde{K}$. This means that the orthogonal distribution is integrable, and holds if and only if $\left(* D^{g} K\right)(K, \widetilde{K})$ and $\left(* D^{g} \widetilde{K}\right)(K, \widetilde{K})$ are both zero. In four dimensions, these are both scalars, the twist scalars. 
We first collect some simple facts about commuting Killing fields.

Lemma 3.1. Suppose $K, \widetilde{K}$ are commuting Killing fields of a 4dimensional metric $g$ and let $\Psi=\left(D^{g} K\right)^{-}, \widetilde{\Psi}=\left(D^{g} \widetilde{K}\right)^{-}$. Then:

(i) $\left(D^{g} K\right)(K, \widetilde{K})=0$ and $\left(D^{g} \widetilde{K}\right)(K, \widetilde{K})=0$.

(ii) $d\left(|\widetilde{\Psi}|^{2}\right)(K)=0$ and $d\left(|\Psi|^{2}\right)(\widetilde{K})=0$.

Proof. (i) Since $[K, \widetilde{K}]=0$, we have $\left(D^{g} K\right)(\widetilde{K}, \cdot)=\left(D^{g} \widetilde{K}\right)(K, \cdot)$. The results follow by contracting with $K$ and $\widetilde{K}$ respectively.

(ii) Since $\mathcal{L}_{K} g=0$ and $\mathcal{L}_{K} \widetilde{K}=0$, we have $\mathcal{L}_{K} D^{g} \widetilde{K}=0$ and hence $\mathcal{L}_{K}(\widetilde{\Psi})=0$. We deduce that $d\left(|\widetilde{\Psi}|^{2}\right)(K)=0$, and, in the same way, $d\left(|\Psi|^{2}\right)(\widetilde{K})=0$.

q.e.d.

Combining this with Proposition 2.1 yields the surface-orthogonality.

Proposition 3.2. Let $g$ be a selfdual Einstein metric of nonzero scalar curvature, and suppose $K, \widetilde{K}$ are linearly independent commuting Killing fields. Then the orthogonal distribution to $\langle\{K, \widetilde{K}\}\rangle$ is integrable.

Proof. Contracting the formula of Proposition 2.1 (i) with $\Psi$, we obtain $\operatorname{grad}_{g}|\Psi|^{2}=s^{g} \Psi(K)$. Now $d\left(|\Psi|^{2}\right)(\widetilde{K})=0$ and $s^{g}$ is nonzero, so we deduce that $\langle\Psi(K), \widetilde{K}\rangle=0$. This implies that $\left(* D^{g} K\right)(K, \widetilde{K})=$ $\left(D^{g} K\right)(K, \widetilde{K})$ which vanishes by the above lemma. A similar argument shows that the other twist scalar also vanishes.

q.e.d.

In the zero scalar curvature case, the twist scalars are constant, but they need not vanish.

\section{Scalar-flat Kähler metrics and Toda structures}

On any Riemannian 4-manifold $(M, g)$, the twistor equation has a geometric interpretation due to Pontecorvo.

Proposition 4.1 ([33]). Suppose that $\Psi$ is a section of $\mathfrak{s o}_{-}(T M)$ satisfying the equation $D_{X}^{g} \Psi=(\gamma \triangle X)^{-}$for all vector fields $X$, where $\gamma$ is a 1-form, and write, on the open set where $\Psi$ is nonzero, $\Psi=f J$ where $J^{2}=-1$.

Then $\left(f^{-2} g, J\right)$ is a (negatively oriented) Kähler metric. In particular the antiselfdual almost complex structure $J$ is integrable.

Proof. Contracting the equation with $\Psi$, we deduce that $\gamma=-2 J d f$. Hence $f D_{X}^{g} J+d f(X) J=-2(J d f \Delta X)^{-}$, which may be rewritten $D_{X}^{g} J$ 
$+\left(f^{-1} d f \Delta X\right) \circ J-J \circ\left(f^{-1} d f \Delta X\right)=0$. This means that $J$ is parallel with respect to the Levi-Civita connection of $f^{-2} g$. $\quad$ q.e.d.

Since the Kähler form of a negatively oriented Kähler metric $\hat{g}$ is parallel, it is a twistor with respect to $\hat{g}$. However, the twistor equation is conformally invariant if $\Psi$ has weight 1 (the other component of the covariant derivative of $\Psi$, the divergence, is equivalently the exterior derivative of the associated 2-form, and so it is conformally invariant if $\Psi$ has weight -2$)$. This means that all compatible Kähler metrics arise from twistors.

If $(M, g)$ is selfdual, then $\left(f^{-2} g, J\right)$ is a scalar-flat Kähler metric [20]. Hence on a selfdual manifold, compatible scalar-flat Kähler metrics are determined locally by solutions of a linear differential equation.

Now suppose that $K$ is a Killing field of $g$, and that $\mathcal{L}_{K} \Psi=0$. Then $K$ is a holomorphic Killing field of the scalar-flat Kähler metric $\left(f^{-2} g, J\right)$. LeBrun [29] shows that such a scalar-flat Kähler metric $g_{J}$ is locally of the form

$$
g_{J}=w e^{u}\left(d x^{2}+d y^{2}\right)+w d z^{2}+w^{-1}(d t+A)^{2},
$$

where $\partial_{t}$ is the Killing field, $u$ is a solution of the $\mathrm{SU}(\infty)$ Toda equation $u_{x x}+u_{y y}+\left(e^{u}\right)_{z z}=0$, and $w$ is a solution of its linearization $w_{x x}+$ $w_{y y}+\left(e^{u} w\right)_{z z}=0$, which is the compatibility condition for the local existence of $A$ with $d A=w_{x} d y \wedge d z-w_{y} d x \wedge d z+\left(e^{u} w\right)_{z} d x \wedge d y$. The scalar-flat Kähler metric is hyperkähler if and only if $u_{z}$ is a multiple of $w$, when LeBrun's construction reduces to that of Boyer and Finlay [6] (or the Gibbons-Hawking Ansatz if $u_{z}=0$ ).

A geometrical interpretation of this construction is obtained by relating it to the Jones-Tod correspondence [25]. Given a selfdual space $M$ with a nonvanishing conformal vector field $K$, the local quotient of $M$ by $K$ is a 3 -dimensional Einstein-Weyl space $B$ - recall that this is a conformal manifold equipped with a torsion-free conformal connection $D$ (a Weyl connection) such that the symmetric trace-free part of the Ricci curvature of $D$ vanishes [10]. Weyl connections on a conformal manifold form an affine space modelled on the space of 1-forms. In the Jones-Tod construction, there is a unique compatible metric for which $K$ is a vector field of constant length, and $D$ differs from the Levi-Civita connection of the quotient metric by a multiple of the twist of $K$ (which descends to a 1 -form on $B$ ).

Conversely, given an Einstein-Weyl space $B$, selfdual spaces $M$ with a conformal vector field fibering over $B$ are locally determined by solu- 
tions of the abelian monopole equation $* D_{W}=d A$ (where $w$ is a section of $L^{-1}$, i.e., a scalar of weight -1 , and $A$ is a 1 -form).

In explicit terms, a Weyl structure may be specified by a choice of representative metric $h$ and a 1 -form $\omega$ such that $D h=-2 \omega \otimes h$. The Einstein-Weyl structure in LeBrun's construction is given by

$$
\begin{aligned}
& h=e^{u}\left(d x^{2}+d y^{2}\right)+d z^{2} \\
& \omega=-u_{z} d z .
\end{aligned}
$$

An Einstein-Weyl space which can be written in this form, for some solution $u$ of the $\mathrm{SU}(\infty)$ Toda equation, is said to be Toda. These Einstein-Weyl spaces were introduced by Ward [40].

The reduction to three dimensions of the twistor equation for $\Psi$ governing scalar-flat Kähler metrics gives a linear description of compatible 'Toda structures' on an Einstein-Weyl space [9].

Definition 4.2. A Toda structure on an Einstein-Weyl is a section $\mathcal{X}$ of $L^{-1 / 2} \otimes T B$ such that $D \mathcal{X}=\sigma$ id for some section $\sigma$ of $L^{-1 / 2}$. In other words, $\mathcal{X}$ is a weighted vector field with tracelike covariant derivative.

Using work of Tod [38] (see also [11]), it was shown in [9] that if $\mathcal{X}$ is a nonvanishing Toda structure, then in the gauge $(h, \omega)$ with $|\mathcal{X}|=1$, called the LeBrun-Ward gauge, the Einstein-Weyl space with is of the form (4.1), for some solution of the $\mathrm{SU}(\infty)$ Toda equation, and $\mathcal{X}=\partial_{z}$.

We end this section by stating the crucial result of Tod [39] which characterizes selfdual Einstein metrics $g$ of nonzero scalar curvature with a Killing field $K$. Proposition 2.1 shows that these admit a solution $\Psi$ of the twistor equation, and it is clear that $\mathcal{L}_{K} \Psi=0$.

Proposition 4.3 ([39]). Let $g$ be a selfdual Einstein metric of nonzero scalar curvature with a Killing field $K$. Then $g$ is locally isometric to $z^{-2} g_{J}$, where $g_{J}$ is a scalar-flat Kähler metric arising from LeBrun's construction with $w=2-z u_{z}$. Conversely, on any Toda Einstein-Weyl space, $2-z u_{z}$ is a solution of the abelian monopole equation and $z^{-2} g_{J}$ is Einstein.

Note that a Toda structure only determines $z$ up to translation, so this construction gives a one parameter family of selfdual Einstein metrics over any Toda Einstein-Weyl space. However, it is very difficult to obtain explicit solutions of the $\mathrm{SU}(\infty)$ Toda equation! In this paper, we are, in effect, exploiting some implicit solutions of the $\mathrm{SU}(\infty)$ Toda equation found by Ward [40]. 


\section{The Joyce Ansatz and Einstein-Weyl spaces}

In [26], Joyce studied selfdual spaces with a surface-orthogonal action of the 2-torus by conformal transformations and constructed selfdual conformal metrics on connected sums of complex projective planes. To do this, he first considered the local problem, and showed how selfdual conformal metrics with a pair of surface-orthogonal commuting conformal vector fields are generically determined by two solutions of a linear equation for a spinor field $\Phi$ on the hyperbolic plane $\mathcal{H}^{2}$.

On the other hand, in [40], Ward gave examples of Toda EinsteinWeyl spaces by taking quotients of Gibbons-Hawking metrics constructed from axisymmetric harmonic functions (AHFs) on $\mathbb{R}^{3}$. These spaces were studied further in [9], where it was shown that they are determined by a single solution $\Phi$ of the same linear equation on $\mathcal{H}^{2}$.

We refer to this equation for $\Phi$, which can be written $\bar{\partial} \Phi=\frac{1}{2} \bar{\Phi}$, as the Joyce equation.

The two constructions can be related using the Jones-Tod correspondence. To do this in a symmetrical manner, we view the span of two commuting conformal vector fields as a 2-dimensional linear family $K^{s}, s \in \mathbb{V}$, where $\mathbb{V}$ is a 2-dimensional real vector space on which we fix an area form (i.e., $\wedge^{2} \mathbb{V}=\mathbb{R}$ ) once and for all. We say that $K^{s}$ is a pencil of conformal vector fields, since the nonzero elements up to scale are parameterized by a real projective line.

We shall call a selfdual conformal manifold with a surface-orthogonal pencil of conformal vector fields a Joyce space. A pencil of solutions $\mathbf{\Phi}=$ $\left(\Phi_{s}\right)$ of the Joyce equation determines a Joyce space. On the other hand, a single solution $\Phi$ of the Joyce equation defines an Einstein-Weyl space with an axial symmetry, i.e., admitting a surface-orthogonal divergencefree conformal vector field $K$ preserving the Weyl connection. We shall see that the pencil of quotients of a Joyce space are the Einstein-Weyl spaces with an axial symmetry defined by the components $\Phi_{s}$ of $\boldsymbol{\Phi}$ (in fact this pencil is parameterized by $\mathbb{V}^{*}$, not $\mathbb{V}$, which is why it is convenient to fix an area form on $\mathbb{V}$ ). First let us summarize the two constructions.

Proposition 5.1. Suppose that $\left(N, g_{N}\right)$ is a hyperbolic 2-manifold with a spinor bundle $\mathcal{W}$, i.e., a real rank 2 vector bundle with a complex structure such that $\mathcal{W} \otimes_{\mathbb{C}} \mathcal{W}=T N$. Let $g_{\mathcal{W}}$ be the induced Hermitian metric on $\mathcal{W}$.

(i) [9] Suppose that $\Phi \in \mathrm{C}^{\infty}\left(N, \mathcal{W}^{*}\right)$ is a solution of the Joyce equation 
which is nonvanishing on an open subset $U$ of $N$, and let $\pi: B \rightarrow U$ be a flat principal $S^{1}$ - or $\mathbb{R}$-bundle with fibre coordinate $\psi$. Then

$$
\begin{aligned}
g & =|\Phi|^{2} \pi^{*} g_{N}+d \psi^{2} \\
\omega & =\Phi^{2} /|\Phi|^{2}
\end{aligned}
$$

is an Einstein-Weyl space with an axial symmetry. Conversely any connected Einstein-Weyl space with an axial symmetry is either flat with translational symmetry, or is locally isomorphic to one of these.

(ii) [26] Suppose that $\boldsymbol{\Phi} \in \mathrm{C}^{\infty}\left(N, \mathcal{W}^{*}\right) \otimes \mathbb{V}$ is a pencil of solutions of the Joyce equation which induces a positive isomorphism $\mathcal{W}_{x} \rightarrow \mathbb{V}$ of real vector spaces for all $x$ in an open subset $U$ of $N$, and let $\pi: M \rightarrow U$ be a flat principal $\mathbb{V} / \Lambda$-bundle where $\Lambda$ is a discrete subgroup of $(\mathbb{V},+)$. Then the conformal class of the metric

$$
\pi^{*} g_{N}+g_{\mathcal{W}}\left(\mathbf{\Phi}^{-1}(\cdot), \boldsymbol{\Phi}^{-1}(\cdot)\right)
$$

(where we identify $T M$ with $\pi^{*} T N \oplus(M \times \mathbb{V})$, using the principal connection) is a Joyce space. Conversely, any connected Joyce space is either locally conformally hyperkähler with a pencil of triholomorphic Killing fields, or is locally isomorphic to one of these.

(For local questions we may as well take $N=\mathcal{H}^{2}, B=U \times \mathbb{R}$ and $M=U \times \mathbb{V}$.)

The conventions used to identify $\bar{\partial} \Phi$ with $\frac{1}{2} \bar{\Phi}$ in the Joyce equation are crucial. If the curvature of the hyperbolic metric is -1 , the isomorphism from $\overline{\mathcal{W}}^{*}$ (which is just $\mathcal{W}^{*}$ with the opposite complex structure) to $\overline{T^{*} N} \otimes_{\mathbb{C}} \mathcal{W}^{*}$ must have norm 1 (as an isomorphism of real vector bundles) with respect to the Hermitian metrics $g_{\mathcal{W}}$ and $g_{N}$.

To clarify this, we follow Joyce by giving an explicit and purely real interpretation of the Joyce equation. We take $N$ to be the hyperbolic plane $\mathcal{H}^{2}$ and introduce half-space coordinates $(\rho>0, \eta)$, so that $g_{\mathcal{H}^{2}}=$ $\left(d \rho^{2}+d \eta^{2}\right) / \rho^{2}$. The metric on $\mathcal{W}$ then has the form $g_{\mathcal{W}}=\mu_{0}^{2}+\mu_{1}^{2}$ where $\mu_{0}^{2}-\mu_{1}^{2}=d \rho / \rho$ and $2 \mu_{0} \mu_{1}=d \eta / \rho$ is the identification of $S_{0}^{2} \mathcal{W}^{*}$ with $T^{*} N$.

We write the Joyce equation for the components $\Phi=A_{0} \mu_{0}+A_{1} \mu_{1}$ with respect to this orthonormal frame. The Levi-Civita connection of the hyperbolic metric induces a Hermitian connection $\nabla$ on $\mathcal{W}$ and it is straightforward to compute the connection coefficients $\nabla \mu_{0}=-\frac{d \eta}{2 \rho} \otimes \mu_{1}$ and $\nabla \mu_{1}=\frac{d \eta}{2 \rho} \otimes \mu_{0}$. Hence

$$
\bar{\partial} \Phi=\left(\rho\left(A_{0}\right)_{\rho}+\rho\left(A_{1}\right)_{\eta}-\frac{1}{2} A_{0}\right) \mu_{0}-\left(\rho\left(A_{1}\right)_{\rho}-\rho\left(A_{0}\right)_{\eta}-\frac{1}{2} A_{1}\right) \mu_{1},
$$


which equals $\frac{1}{2}\left(A_{0} \mu_{0}+A_{1} \mu_{1}\right)$ if and only if

$$
\begin{aligned}
& \left(A_{0}\right)_{\rho}+\left(A_{1}\right)_{\eta}=A_{0} / \rho \\
& \left(A_{0}\right)_{\eta}-\left(A_{1}\right)_{\rho}=0 .
\end{aligned}
$$

By solving one of these equations, we can reduce the Joyce equation to an equation for a single function. The most obvious way to do this is to use the second equation to set $A_{0}=G_{\rho}$ and $A_{1}=G_{\eta}$ so that the first equation becomes $G_{\rho \rho}+G_{\eta \eta}=G_{\rho} / \rho$. If we set $G=\rho^{1 / 2} F$ then $F$ is an eigenfunction of the Laplacian on $\mathcal{H}^{2}$ with eigenvalue $3 / 4$.

Alternatively we can use the first equation to put $A_{0}=-\rho V_{\eta}$ and $A_{1}=\rho V_{\rho}$, so that the second equation becomes $\rho V_{\eta \eta}+\left(\rho V_{\rho}\right)_{\rho}=0$. This means that $V$ is an AHF on $\mathbb{R}^{3}$ with metric $d \rho^{2}+d \eta^{2}+\rho^{2} d \theta^{2}$.

By construction, the equation for $F$ is the integrability condition for $V$ and vice-versa, so the relation between $F$ and $V$ is a simple example of a Bäcklund transformation.

Suppose now that we have two solutions $A_{0} \mu_{0}+A_{1} \mu_{1}$ and $B_{0} \mu_{0}+$ $B_{1} \mu_{1}$ of the Joyce equation. The corresponding Joyce space has a compatible metric

$$
g_{0}=\left(A_{0} B_{1}-A_{1} B_{0}\right) g_{\mathcal{H}^{2}}+\frac{\left(A_{0} d \phi-B_{0} d \psi\right)^{2}+\left(A_{1} d \phi-B_{1} d \psi\right)^{2}}{A_{0} B_{1}-A_{1} B_{0}}
$$

Via the Jones-Tod correspondence [25], the quotient by $\partial_{\phi}$ is an Einstein-Weyl space, and we can compute it by rediagonalizing $g_{0}$ and rescaling by $\left(A_{0}^{2}+A_{1}^{2}\right) /\left(A_{0} B_{1}-A_{1} B_{0}\right)$ to give

$$
\left(A_{0}^{2}+A_{1}^{2}\right) g_{\mathcal{H}^{2}}+d \psi^{2}+\left(\frac{A_{0}^{2}+A_{1}^{2}}{A_{0} B_{1}-A_{1} B_{0}}\right)^{2}\left(d \phi-\frac{\left(A_{0} B_{0}+A_{1} B_{1}\right) d \psi}{A_{0}^{2}+A_{1}^{2}}\right)^{2} .
$$

In this form, we can read off the Einstein-Weyl space $\left(g_{B}, \omega_{B}\right)$ and abelian monopole $(w, A)$, using the abelian monopole equation $*\left(d_{W}-\right.$ $\left.\omega_{B} W\right)=d A$ to compute $\omega_{B}$. The result is:

$$
\begin{aligned}
g_{B} & =\left(A_{0}^{2}+A_{1}^{2}\right) g_{\mathcal{H}^{2}}+d \psi^{2}, & \omega_{B} & =\frac{2 A_{0} A_{1} d \eta+\left(A_{0}^{2}-A_{1}^{2}\right) d \rho}{\rho\left(A_{0}^{2}+A_{1}^{2}\right)}, \\
w & =\frac{A_{1} B_{0}-A_{0} B_{1}}{A_{0}^{2}+A_{1}^{2}}, & A & =-\frac{A_{0} B_{0}+A_{1} B_{1}}{A_{0}^{2}+A_{1}^{2}} d \psi .
\end{aligned}
$$

Hence, as expected, the result is the Einstein-Weyl space constructed from the solution $A_{0} \mu_{0}+A_{1} \mu_{1}$ of the Joyce equation. However, we also 
see explicitly how the second solution $B_{0} \mu_{0}+B_{1} \mu_{1}$ of the Joyce equation determines an abelian monopole on this Einstein-Weyl space. In the remainder of this section we discuss the scalar-flat Kähler metrics and Toda structures that will enable us to characterize the case that $\left(M, g_{0}\right)$ is conformally Einstein.

An important point is that any Joyce space admits a family of scalarflat Kähler metrics [26], and any Einstein-Weyl space with an axial symmetry admits a family of Toda structures [9]. In fact, Joyce observes that each point at infinity of $\mathcal{H}^{2}$ determines a scalar-flat Kähler metric in the conformal class on $M$ : more precisely, for any half-space coordinates $(\rho, \eta)$ on $\mathcal{H}^{2}$, the metric $\rho g_{0}$ is scalar-flat Kähler. By Pontecorvo's work [33], this means that $M$ has a 2-dimensional linear family of solutions of the twistor equation, which we parameterize by a 2-dimensional real vector space $\mathbb{W}$.

Since these scalar-flat Kähler metrics are invariant under the entire pencil of conformal vector fields, LeBrun's work [29] shows that each scalar-flat Kähler metric determines a Toda structure on each quotient Einstein-Weyl space. This fits together with a further characterization of Einstein-Weyl spaces with an axial symmetry [9]: they are precisely the Einstein-Weyl spaces admitting a pencil of Toda structures. These Toda structures are parameterized by the same 2 -dimensional real vector space $\mathbb{W}$, and are obtained from a choice of half-space coordinates $(\rho, \eta)$ on $\mathcal{H}^{2}$ as follows.

First introduce the functions $G, V$ with $G_{\rho}=A_{0}=-\rho V_{\eta}$ and $G_{\eta}=$ $A_{1}=\rho V_{\rho}$. (Note that $G$ is determined by $V$ up to translation: one way to define $G$ is to choose an AHF $U$ with $U_{\eta}=V$, so that $G=\rho U_{\rho}$.) Then, after a conformal rescaling by $\rho^{2}$, the Einstein-Weyl structure may be written in the form (4.1),

$$
h=\rho^{2}\left(d V^{2}+d \psi^{2}\right)+d G^{2}, \quad \omega=\frac{2 V_{\eta}}{\rho^{2}\left(V_{\rho}^{2}+V_{\eta}^{2}\right)} d G,
$$

where we set $x=V, y=\psi, z=G$, and $e^{u}=\rho^{2}$.

We know that $u_{z}$ is an abelian monopole giving rise to hyperkähler metric [6], while the Einstein metrics we seek are obtained from a $2-z u_{z}$ monopole (once we have fixed the Toda structure and the translational freedom in the $z$ coordinate). The conformal rescaling by $\rho^{2}$ multiplies the abelian monopoles $w$ by (a constant multiple of) $1 / \rho$, so in the 
original gauge, these monopoles may be written

$$
\begin{aligned}
\frac{\rho}{2} u_{z} & =\frac{A_{0}}{A_{0}^{2}+A_{1}^{2}} \\
\frac{\rho}{2}\left(2-z u_{z}\right) & =\frac{\rho\left(A_{0}^{2}+A_{1}^{2}\right)-G A_{0}}{A_{0}^{2}+A_{1}^{2}} .
\end{aligned}
$$

Clearly (from (5.3)) the first of these is the abelian monopole associated to the solution $\mu_{1}$ of the Joyce equation. The second turns out to be associated to the solution $B_{0} \mu_{0}+B_{1} \mu_{1}$ with $B_{0}=\rho A_{1}-\eta A_{0}$ and $B_{1}=G-\rho A_{0}-\eta A_{1}$. This latter formula is rather mysterious at present, though one easily checks that it does satisfy the Joyce equation, and that $A_{1} B_{0}-A_{0} B_{1}=\rho\left(A_{0}^{2}+A_{1}^{2}\right)-G A_{0}$. The origin of this transformation of the Joyce equation will be explained later.

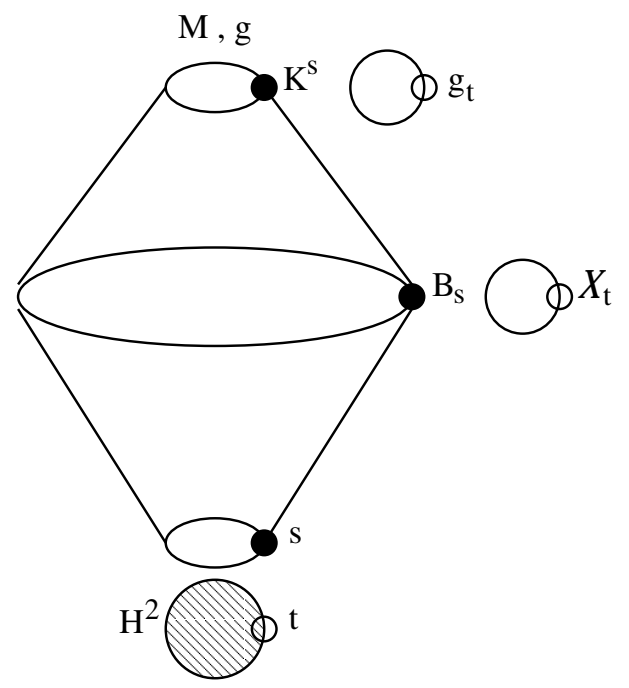

Figure 1.

Let us now summarize the discussion of this section (see Figure 1). First, each solution $\Phi$ of the Joyce equation determines an EinsteinWeyl space with an axial symmetry. A pencil of solutions $\boldsymbol{\Phi}=\left(\Phi_{s}\right)$ defines a pencil of Einstein-Weyl spaces $B_{s}$ and a selfdual space $M$ with a surface-orthogonal pencil of conformal vector fields $K^{s}$, having the Einstein-Weyl spaces $B_{s}$ as quotients. On the other hand, any such selfdual space $M$ has a pencil of compatible scalar-flat Kähler metrics $g_{t}$ and these determine a pencil of Toda structures $\mathcal{X}_{t}$ on each quotient Einstein-Weyl space $B_{s}$. The choice of a scalar-flat Kähler metric or 
Toda structure is given, up to homothety, by a point $t$ at infinity on the hyperbolic plane $\mathcal{H}^{2}$. We can fix the homothety freedom by introducing half-space coordinates for the given point at infinity. We shall see in the next section how to regard points at infinity of $\mathcal{H}^{2}$ as a pencil.

\section{Spin geometry of the hyperbolic plane}

The hyperbolic plane $\mathcal{H}^{2}$ is most naturally described as the space of timelike lines in a 3-dimensional Lorentzian vector space: it inherits a Riemannian metric from the observation that each timelike line meets the hyperboloid of two sheets at two points, one in each sheet; thus we may identify $\mathcal{H}^{2}$ with one of these sheets, with the induced metric. Planar models (such as the Poincaré disc or half-plane) are obtained by introducing coordinates (pairs of real-valued functions with independent differentials) on $\mathcal{H}^{2}$.

In this section we describe the geometry of spinors on the hyperbolic plane by equipping the Lorentzian vector space with some extra structure, following Iversen [24].

Let $\mathbb{W}$ be a 2 -dimensional real vector space with an area form $\varepsilon$ (so $\left.\wedge^{2} \mathbb{W}=\mathbb{R}\right)$. Then $S^{2} \mathbb{W}$ is a $(++-)$ Lorentzian vector space with metric $\left\langle v_{1} v_{2}, w_{1} w_{2}\right\rangle=-\varepsilon\left(v_{1}, w_{1}\right) \varepsilon\left(v_{2}, w_{2}\right)-\varepsilon\left(v_{1}, w_{2}\right) \varepsilon\left(v_{2}, w_{1}\right)$. Alternatively, we can use $\varepsilon$ to identify $S^{2} \mathbb{W}$ with $\mathfrak{s l}(\mathbb{W})$, the Lie algebra of traceless linear maps $A: \mathbb{W} \rightarrow \mathbb{W}$, so that the Lorentzian quadratic form is $A \mapsto$ - $\operatorname{det} A$, i.e., the timelike vectors have positive determinant.

This linear algebra is a manifestation of the well-known isomorphism between $\operatorname{Spin}(2,1)$ and $\operatorname{SL}(2, \mathbb{R}): \mathbb{W}$ is the space of spinors of $S^{2} \mathbb{W}$. The hyperboloid of two sheets is the surface $\operatorname{det} A=1$ and so we use this description to identify $\mathcal{H}^{2}$ with the positive definite elements of $S^{2} \mathbb{W}$ of determinant one.

In more concrete terms, choosing a unimodular basis of $\mathbb{W}$, we can parameterize $\mathcal{H}^{2}$ in $S^{2} \mathbb{W}$ by the matrices

$$
A(\rho, \eta)=\frac{1}{\rho}\left[\begin{array}{cc}
1 & \eta \\
\eta & \rho^{2}+\eta^{2}
\end{array}\right]
$$

Note that the corresponding traceless matrices are obtained by multiplying these symmetric matrices by $J=\left[\begin{array}{cc}0 & -1 \\ 1 & 0\end{array}\right]$, which has determinant 
one. We easily compute that

$$
d A=\frac{d \rho}{\rho}\left[\begin{array}{cc}
-1 / \rho & -\eta / \rho \\
-\eta / \rho & \left(\rho^{2}-\eta^{2}\right) / \rho
\end{array}\right]+\frac{d \eta}{\rho}\left[\begin{array}{cc}
0 & 1 \\
1 & 2 \eta
\end{array}\right]
$$

so that $\quad\langle d A, d A\rangle=\frac{d \rho^{2}+d \eta^{2}}{\rho^{2}}$.

Thus $\rho$ and $\eta$ are functions on $\mathcal{H}^{2}$ identifying it with the standard halfspace model of the hyperbolic plane. They are only defined once we have chosen the unimodular basis of $\mathbb{W}$.

The advantage of this model of the hyperbolic plane is that spinors are easy to handle. Indeed we can identify $\mathcal{H}^{2} \times \mathbb{W}$ with the spinor bundle by noting that for each $A \in \mathcal{H}^{2}, A^{-1}$ is, by definition, a positive definite unimodular inner product on $\mathbb{W}$, and this equips $\mathcal{W}=\mathcal{H}^{2} \times \mathbb{W}$ with a metric. The induced complex structure evidently satisfies $\mathcal{W}^{2}=T \mathcal{H}^{2}$, since $\mathcal{W}_{A}^{2}$ consists of the symmetric elements in $\mathbb{W} \otimes_{\mathbb{R}} \mathbb{W}$ which are traceless with respect to the inner product $A^{-1}$, i.e., orthogonal to $A$, and this is the tangent plane $T_{A} \mathcal{H}^{2}$.

In terms of a half-space model, a frame for $\mathcal{W}$ is given by the vectors

$$
m_{0}=\left[\begin{array}{c}
0 \\
\sqrt{\rho}
\end{array}\right], \quad m_{1}=\left[\begin{array}{l}
1 / \sqrt{\rho} \\
\eta / \sqrt{\rho}
\end{array}\right]
$$

with dual frame

$$
\mu_{0}=\left[\begin{array}{c}
-\eta / \sqrt{\rho} \\
1 / \sqrt{\rho}
\end{array}\right], \quad \mu_{1}=\left[\begin{array}{c}
\sqrt{\rho} \\
0
\end{array}\right]
$$

One easily sees that these are orthogonal and of unit length with respect to $A$, and that

$$
m_{0}^{2}-m_{1}^{2}=d A\left(\rho \partial_{\rho}\right), \quad 2 m_{0} m_{1}=d A\left(\rho \partial_{\eta}\right) .
$$

We refer to the "constant" sections of $\mathcal{W}=\mathcal{H}^{2} \times \mathbb{W}$ as twistors they are certainly not parallel with respect to the induced Hermitian connection on $\mathcal{W}$, since the hyperbolic metric is not flat. We shall not need to discuss what equation they satisfy, since we have an explicit description of them in terms of the orthonormal frame $m_{0}, m_{1}$ : they are the sections of the form

$$
\left[\begin{array}{l}
a \\
b
\end{array}\right]=-\frac{a \eta-b}{\sqrt{\rho}} m_{0}+a \sqrt{\rho} m_{1},
$$


for $a, b \in \mathbb{R}$. In particular, the norm of $\left[\begin{array}{l}a \\ b\end{array}\right]$ is $\sqrt{a^{2} \rho^{2}+(a \eta-b)^{2}} / \sqrt{\rho}$.

For a geometric interpretation, note that $P(\mathbb{W})$ is the space of null lines in $S^{2} \mathbb{W}$, which are the points at infinity of the hyperbolic plane. A point at infinity identifies the hyperbolic plane with the half-space model. The inverse square norm of a twistor gives a ' $\rho$ ' coordinate for this half-space model. Evidently, the ' $\rho$ ' coordinate determines the twistor up to a sign.

\section{A tale of two pencils}

We have introduced two pencils, parameterized by the vector spaces $\mathbb{V}$ and $\mathbb{W}$. The first vector space $\mathbb{V}$ parameterizes the pencils of:

- conformal vector fields on the selfdual space $M$ with torus symmetry;

- Einstein-Weyl spaces with an axial symmetry arising as quotients of $M$;

- solutions of the Joyce equation determining these Einstein-Weyl spaces.

The second vector space $\mathbb{W}$ parameterizes the pencils induced by (deprojectivized) points at infinity of the hyperbolic plane, i.e., the pencils of:

- compatible scalar-flat Kähler metrics on $M$;

- Toda structures on the Einstein-Weyl spaces;

- twistors on the hyperbolic plane.

The idea now is that when the selfdual space $M$ is given by a selfdual Einstein metric $g$ of nonzero scalar curvature, these pencils are the same:

- each Killing field determines a scalar-flat metric;

- each Einstein-Weyl space has one of its Toda structures distinguished;

and therefore there must be a field on $\mathcal{H}^{2}$ which determines a linear map from twistors to solutions of the Joyce equation, and the resulting linear family of solutions is the pencil defining the underlying conformal structure of the Einstein metric. In this section we prove that this field is the eigenfunction $F$. The heart of the argument is the following result: 
Proposition 7.1. Let $F$ be an eigenfunction of the Laplacian on the hyperbolic plane with eigenvalue $3 / 4$ and let $\varphi$ be a twistor. Then $\Phi=\frac{1}{2} F b \varphi+d F \cdot \varphi$ is a solution of the Joyce equation, where $d F \cdot \varphi$ denotes the natural pairing $T^{*} \mathcal{H}^{2} \otimes \mathcal{W} \rightarrow \mathcal{W}^{*}$.

Proof. Given a twistor $\varphi$, we can use the freedom in the choice of half-space model to set $\varphi=m_{0} / \sqrt{\rho}$. In these half-space coordinates $F$ satisfies the equation

$$
F_{\rho \rho}+F_{\eta \eta}=\frac{3 F}{4 \rho^{2}}
$$

Since $d F=\rho F_{\rho}\left(\mu_{0}^{2}-\mu_{1}^{2}\right)+2 \rho F_{\eta} \mu_{0} \mu_{1}$ direct calculation yields $\Phi=$ $\left(\rho^{1 / 2} F\right)_{\rho} \mu_{0}+\left(\rho^{1 / 2} F\right)_{\eta} \mu_{1}$, so $\Phi$ satisfies the Joyce equation. q.e.d.

Proof of Theorem 1.1. Suppose that $g$ is a selfdual Einstein metric of nonzero scalar curvature on $M$ with two commuting Killing fields. Let us review what we have proven so far about $g$. Firstly, by Proposition 3.2, the Killing fields are surface orthogonal, and therefore, by [26], the conformal class of $g$ is a Joyce space. (If $g$ is conformally hyperkähler, it must be conformally flat, i.e., locally isometric to $S^{4}$ or $\mathcal{H}^{4}$, but then the Killing fields of $g$ cannot all be triholomorphic with respect to the flat hyperkähler metric.)

The quotient of $g$ by one of its Killing fields is an Einstein-Weyl space $B$ with an abelian monopole $w$ [25]. The work of Tod [39] shows that the choice of Killing field determines a compatible scalar-flat Kähler metric on $M$, a Toda structure on $B$, and a coordinate $z$ on $B$ such that the monopole $w$ is $2-z u_{z}$. On the other hand, we showed in Section 5 that $B$ is an Einstein-Weyl space with an axial symmetry. Since the Toda structure is invariant under this symmetry, it is one of the Toda structures determined by a point at infinity on $\mathcal{H}^{2}$. Introducing compatible half-space coordinates we may write the solution of the Joyce equation corresponding to this Einstein-Weyl space as $\Phi=A_{0} \mu_{0}+A_{1} \mu_{1}$ and then $z=G$ for some function $G$ on $\mathcal{H}^{2}$ with $G_{\rho}=A_{0}$ and $G_{\eta}=A_{1}$.

Now set $F=\rho^{-1 / 2} G$. Then $F_{\rho \rho}+F_{\eta \eta}=\frac{3}{4} F / \rho^{2}$ and $\Phi$ is obtained by applying $F$ to the twistor $m_{0} / \sqrt{\rho}$ as in Proposition 7.1. On the other hand, applying $F$ to $\left(-\eta m_{0}+\rho m_{1}\right) / \sqrt{\rho}$ yields the solution $\widetilde{\Phi}=$ $\left(\rho A_{1}-\eta A_{0}\right) \mu_{0}+\left(G-\rho A_{0}-\eta A_{1}\right) \mu_{1}$, which is precisely the solution needed to construct the $2-z u_{z}$ monopole on $B$.

Hence $F$ generates the pencil of solutions of the Joyce equation yielding the underlying conformal structure of the selfdual Einstein metric $g$. The distinguished scalar-flat Kähler metric is $\rho g_{0}$ and rescaling this 
by $1 / z^{2}$, according to Proposition 4.3 , we recover the selfdual Einstein metric $F^{-2} g_{0}$. The explicit formula (1.1) is obtained from (5.1) by direct substitution.

As we remarked in the introduction, the reader who is not convinced that we really have encoded the entire selfdual Einstein condition in the construction can easily verify this directly. Such calculations amount to reproving Tod's result [39] in this special case.

q.e.d.

In the next section we shall be able to obtain a better understanding of formula (1.1) after studying the Swann bundle. We will also indicate there how to check directly that the metric is selfdual and Einstein.

\section{The Swann bundle}

The Swann bundle $\mathcal{U}(M)$ of a selfdual Einstein manifold $(M, g)$ with nonzero scalar curvature is defined to be the principal $\mathrm{CO}(3)$-bundle of conformal frames of $\wedge^{2} T_{-}^{*} M$. In [36], Swann showed how to define a canonical (pseudo-)hyperkähler metric on a similar bundle over any quaternion-kähler manifold.

The hyperkähler structure on $\mathcal{U}(M)$ is obtained as follows. The LeviCivita connection induces a principal $\mathrm{CO}(3)$ connection on $\pi: \mathcal{U}(M) \rightarrow$ $M$. The horizontal bundle of $\mathcal{U}(M)$ is isomorphic to $\pi^{*} T M$, which has three tautological 2-forms (determined by the frame of $\wedge^{2} T_{-}^{*} M$ at each point of $\mathcal{U}(M)$ ), whereas the vertical bundle of $\mathcal{U}(M)$ is isomorphic to $\mathcal{U}(M) \times \mathbb{H}$, since $\mathrm{CO}(3) \cong \mathbb{H}^{\times} /\{ \pm 1\}$, and this has a standard triple of 2 -forms. Adding suitable multiples of the horizontal and vertical components gives the three symplectic forms.

In order to describe this more explicitly, identify $\mathcal{U}(M)$ locally with $M \times \mathrm{CO}(3)$ by choosing a frame $\Theta$ of $\wedge^{2} T_{-}^{*} M$. We view $\Theta$ as a 2 -form on $M$ with values in $\operatorname{Im} \mathbb{H}$. The connection on $\wedge^{2} T_{-}^{*} M$ is given by an $\operatorname{Im} \mathbb{H}$-valued 1-form $\omega$ satisfying

$$
d \Theta-\omega \wedge \Theta+\Theta \wedge \omega=0
$$

where $(\omega \wedge \Theta)(X, Y)=\omega(X) \Theta(Y)-\omega(Y) \Theta(X)$ is the usual wedge product of quaternion-valued forms. The selfdual Einstein equation is now

$$
d \omega-\omega \wedge \omega+s \Theta=0
$$

where $s$ is a constant - up to a positive numerical factor it is the scalar curvature of the selfdual Einstein metric $g$. 
Passing to a double cover, we have an $\mathbb{H}$-valued coordinate $q$ given by the projection $M \times \mathbb{H}^{\times} \rightarrow \mathbb{H}^{\times}$. The hyperkähler metric is then

$$
\widetilde{g}=s|q|^{2} g+|d q+q \omega|^{2}
$$

with $\operatorname{Im} \mathbb{H}$-valued Kähler form $\Omega=s q \Theta \bar{q}+(d q+q \omega) \wedge(\overline{d q+q \omega})$. An easy computation gives

$$
\begin{aligned}
d \Omega=d q \wedge(s \Theta-\omega \wedge \omega+d \omega) \bar{q}+q(s \Theta+d \omega-\omega & \wedge \omega) \wedge d \bar{q} \\
+q(s d \Theta+\omega & \wedge d \omega-d \omega \wedge \omega) \bar{q}
\end{aligned}
$$

which vanishes if $g$ is a selfdual Einstein metric.

Let us turn now to the examples of Theorem 1.1. In this case a frame for $\wedge_{-}^{2} T^{*} M$ is given by

$$
\begin{aligned}
\Theta= & \frac{1}{F^{2}}\left(\left(-\frac{1}{4} F^{2}+\rho^{2}\left(F_{\rho}^{2}+F_{\eta}^{2}\right)\right) \frac{d \rho \wedge d \eta}{\rho^{2}}+\alpha \wedge \beta\right) \boldsymbol{i} \\
& +\frac{1}{F^{2}}\left(\left(\rho F_{\rho}+\boldsymbol{i} \rho F_{\eta}\right)(\alpha-\boldsymbol{i} \beta)-\frac{1}{2} F(\alpha+\boldsymbol{i} \beta)\right) \wedge \frac{d \rho-\boldsymbol{i} d \eta}{\rho} \boldsymbol{j}
\end{aligned}
$$

where $\boldsymbol{i}, \boldsymbol{j}, \boldsymbol{k}$ are the imaginary quaternions. A tedious computation is rewarded by a remarkably simple formula for the connection 1-form:

$$
\omega=\frac{1}{F}\left(-\rho F_{\eta} \frac{d \rho}{\rho}+\left(\frac{1}{2} F+\rho F_{\rho}\right) \frac{d \eta}{\rho}\right) \boldsymbol{i}-\frac{1}{F}(\alpha-\boldsymbol{i} \beta) \boldsymbol{j} .
$$

Computing $d \omega-\omega \wedge \omega$, we can check that the metric $g$ is selfdual and Einstein with $s=1$.

Since the construction of the Swann bundle is canonical, the commuting Killing fields of $g$ lift to give commuting trihamiltonian vector fields of $\widetilde{g}$. Now any hyperkähler 8-manifold with two commuting trihamiltonian vector fields is given explicitly by a generalized GibbonsHawking Ansatz [23, 32]: it is isometric to

$$
\Phi_{i j}\left\langle d \boldsymbol{x}_{i}, d \boldsymbol{x}_{j}\right\rangle+\Phi^{-1}{ }_{i j}\left(d t_{i}+A_{i}\right)\left(d t_{j}+A_{j}\right)
$$

where $\left(\Phi_{i j}, A_{i}\right)$ is a solution of a generalized abelian monopole equation on $\mathbb{R}^{2} \otimes \operatorname{Im} \mathbb{H}$, whose coordinates $\left(\boldsymbol{x}_{1}, \boldsymbol{x}_{2}\right)$ are the $\operatorname{Im} \mathbb{H}$-valued momentum maps of the trihamiltonian vector fields $\left(\partial_{t_{1}}, \partial_{t_{2}}\right)$. In more invariant language, the matrix $\underline{\Phi}$ is a section of $S^{2} \mathbb{V}$ over $\mathbb{V}^{*} \otimes \operatorname{Im} \mathbb{H}$, where $\mathbb{V}$ is the 2 -dimensional real vector space of Killing fields. Our notation $\underline{\Phi}=\left(\Phi_{i j}\right)$ is meant to suggest that this matrix is essentially the same object as the pencil $\boldsymbol{\Phi}=\left(\Phi_{s}\right)$ of solutions of the Joyce equation, as we shall see at the end of this section. First, using the nonconstant frame $(\alpha, \beta)$ for $\mathbb{V}=\mathbb{W}^{*}$ (instead of $\left.(d \phi, d \psi)\right)$, we obtain the following result: 
Proposition 8.1. Let $F$ be a local eigenfunction of the Laplacian on $\mathcal{H}^{2}$. Then the hyperkähler metric on the Swann bundle of the associated selfdual Einstein metric is given by the generalized Gibbons-Hawking Ansatz with

$$
\underline{\Phi}=\frac{F}{|q|^{2}}\left(\begin{array}{cc}
\frac{1}{2} F+\rho F_{\rho} & \rho F_{\eta} \\
\rho F_{\eta} & \frac{1}{2} F-\rho F_{\rho}
\end{array}\right)
$$

Furthermore, the Im $\mathbb{H}$-valued momentum maps of $\partial_{\psi}$ and $\partial_{\phi}$ are

$$
\boldsymbol{x}_{\psi}=\frac{q \boldsymbol{k} \bar{q}}{\sqrt{\rho} F} \quad \text { and } \quad \boldsymbol{x}_{\phi}=\frac{q(\eta+\rho \boldsymbol{i}) \boldsymbol{k} \bar{q}}{\sqrt{\rho} F} .
$$

Proof. For the first part we show that $\underline{\Phi}^{-1}$ is the metric on $\mathcal{U}(M) \times \mathbb{W}$ induced by $\widetilde{g}$. Let $Q$ be the matrix $\left(\begin{array}{cc}\frac{1}{2} F-\rho F_{\rho} & -\rho F_{\eta} \\ -\rho F_{\eta} & \frac{1}{2} F+\rho F_{\rho}\end{array}\right)$, so that the selfdual Einstein metric is

$$
g=\frac{1}{F^{2}}\left[\operatorname{det} Q g_{\mathcal{H}^{2}}+\frac{1}{\operatorname{det} Q}(\alpha \beta) Q^{2}\left(\begin{array}{c}
\alpha \\
\beta
\end{array}\right)\right]
$$

Then the metric on the torus induced by $\widetilde{g}=|q|^{2} g+|d q+q \Theta|^{2}$ is

$$
\frac{|q|^{2}}{F^{2} \operatorname{det} Q}(\alpha \beta)\left(\operatorname{det} Q \mathrm{id}+Q^{2}\right)\left(\begin{array}{c}
\alpha \\
\beta
\end{array}\right)
$$

and $\operatorname{det} Q \mathrm{id}+Q^{2}=(\operatorname{tr} Q) Q=F Q$ by the Cayley-Hamilton theorem. This is what we want, because $\underline{\Phi}^{-1}=|q|^{2} Q /(F \operatorname{det} Q)$.

For the momentum maps, we must compute the contraction of the Im $\mathbb{H}$-valued symplectic form $q \Theta \bar{q}+(d q+q \omega) \wedge(d \bar{q}-\omega \bar{q})$ with $\partial_{\psi}$ and $\partial_{\phi}$. The contraction with any vector field $X$ in the torus is $q(\Theta(X)+$ $\omega(X) \omega-\omega \omega(X)) \bar{q}+q \omega(X) d \bar{q}+d q \omega(X) \bar{q}$. It is straightforward to compare this with $d \boldsymbol{x}_{\psi}$ or $d \boldsymbol{x}_{\phi}$ when $X=\partial_{\psi}$ or $\partial_{\phi}$ : in particular note that $\omega\left(\partial_{\psi}\right)=\boldsymbol{k} / \sqrt{\rho} F$ and $\omega\left(\partial_{\phi}\right)=(\eta+\rho \boldsymbol{i}) \boldsymbol{k} / \sqrt{\rho} F$. $\quad$ q.e.

We summarize this discussion of the Swann bundle by the following diagram:

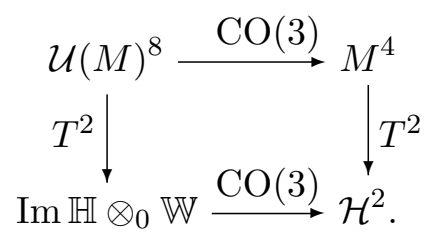


Here we denote by $\operatorname{Im} \mathbb{H} \otimes_{0} \mathbb{W}$ the elements of $\operatorname{Im} \mathbb{H} \otimes \mathbb{W}$ which are not decomposable. In terms of a basis for $\mathbb{W}$, this means the points where the coordinates $\boldsymbol{x}_{1}$ and $\boldsymbol{x}_{2}$ are linearly independent. Clearly the momentum maps $\boldsymbol{x}_{\phi}$ and $\boldsymbol{x}_{\psi}$ are linearly independent.

In order to understand the bottom arrow in this diagram, consider the Grammian map $\operatorname{Im} \mathbb{H} \otimes \mathbb{W} \rightarrow S^{2} \mathbb{W}$ given in components by

$$
\left(\boldsymbol{x}_{1}, \boldsymbol{x}_{2}\right) \mapsto\left[\begin{array}{cc}
\left|\boldsymbol{x}_{1}\right|^{2} & \left\langle\boldsymbol{x}_{1}, \boldsymbol{x}_{2}\right\rangle \\
\left\langle\boldsymbol{x}_{1}, \boldsymbol{x}_{2}\right\rangle & \left|\boldsymbol{x}_{2}\right|^{2}
\end{array}\right]
$$

The determinant of this matrix is $\left|\boldsymbol{x}_{1} \wedge \boldsymbol{x}_{2}\right|^{2}$, which is nonnegative, and vanishes if and only if $\boldsymbol{x}_{1}$ and $\boldsymbol{x}_{2}$ are linearly dependent. Otherwise the matrix is positive definite, and so on dividing by $\left|\boldsymbol{x}_{1} \wedge \boldsymbol{x}_{2}\right|$, we get a well-defined map $\operatorname{Im} \mathbb{H} \otimes_{0} \mathbb{W} \rightarrow \mathcal{H}^{2}$. This map is $\mathrm{CO}(3)$-invariant, where $\mathrm{CO}(3)$ acts diagonally on $\operatorname{Im} \mathbb{H} \otimes \mathbb{W} \cong \operatorname{Im} \mathbb{H} \oplus \operatorname{Im} \mathbb{H}$, and applying it to $\left(\boldsymbol{x}_{\phi}, \boldsymbol{x}_{\psi}\right)$ gives the matrix

$$
\frac{1}{\rho}\left[\begin{array}{cc}
1 & \eta \\
\eta & \rho^{2}+\eta^{2}
\end{array}\right]
$$

so the diagram commutes.

We now attempt to justify our use of the same letter both for solutions of the Joyce equation defining selfdual Einstein metrics, and for generalized monopoles defining their Swann bundles. Given any hyperbolic eigenfunction $F$ on $\mathcal{H}^{2}$, define $\widetilde{F}: S^{2} \mathbb{W}+\mathbb{R}$, where $S^{2} \mathbb{W}$ + denote the space of timelike elements of $S^{2} \mathbb{W}$ (i.e., matrices of positive determinant), by requiring that $\widetilde{F}$ has homogeneity $1 / 2$, i.e., $\widetilde{F}(\lambda v)=\lambda^{1 / 2} \widetilde{F}(v)$, and that $\left.\widetilde{F}\right|_{\mathcal{H}^{2}}=F$. Then $d \widetilde{F}$ is a function on $S^{2} \mathbb{W}+$ with values in $S^{2} \mathbb{W}$ and its matrix with respect to the (homogeneity $1 / 2$ ) orthonormal frame $\left(m_{0}, m_{1}\right)$ of $S^{2} \mathbb{W}_{+} \times \mathbb{W}$ is

$$
\begin{array}{r}
\frac{1}{\sqrt{\operatorname{det} A}}\left(\begin{array}{cc}
\frac{1}{2} F+\rho F_{\rho} & \rho F_{\eta} \\
\rho F_{\eta} & \frac{1}{2} F-\rho F_{\rho}
\end{array}\right) \quad \text { at } \\
A=\frac{\sqrt{\operatorname{det} A}}{\rho}\left[\begin{array}{cc}
1 & \eta \\
\eta & \rho^{2}+\eta^{2}
\end{array}\right] \in S^{2} \mathbb{W}_{+} \cdot
\end{array}
$$

- Pulling $d \widetilde{F}$ back to $\operatorname{Im} \mathbb{H} \otimes_{0} \mathbb{W}$ gives the generalized monopole $\underline{\Phi}$, since $\sqrt{\operatorname{det} A}$ pulls back to $\left|\boldsymbol{x}_{\psi} \wedge \boldsymbol{x}_{\phi}\right|=|q|^{2} / F$.

- Restricting $d \widetilde{F}$ to $\mathcal{H}^{2}$ gives the pencil of solutions $\boldsymbol{\Phi}$ of the Joyce equation and this explains the form of the metric in (1.1). 
Hence $\underline{\Phi}$ and $\boldsymbol{\Phi}$ are different manifestations of the same object. Furthermore, this description in terms of a homogeneity $1 / 2$ function $\widetilde{F}$ gives a natural interpretation of the equation for $F: F=\left.\widetilde{F}\right|_{\mathcal{H}^{2}}$ is a hyperbolic eigenfunction with eigenvalue $3 / 4$ if and only if $\widetilde{F}$ is a (homogeneity $1 / 2$ ) solution of the wave equation.

\section{Quaternion-kähler and hyperkähler quotients}

Our motivation for constructing the Swann bundle of the selfdual Einstein metrics of this paper is to provide an explicit relation between these metrics and the quaternion-kähler quotients of quaternionic projective space $\mathbb{H} P^{m-1}$. In $[16,19]$ Galicki and Lawson defined an analogue of the hyperkähler quotient [23] in quaternion-kähler geometry, in which the quotient of a $4(m-1)$-dimensional quaternion-kähler manifold by a $k$-dimensional group of symmetries is (at least locally) a $4(m-k-1)$ dimensional quaternion-kähler manifold.

This is of interest here, because quaternion-kähler quotients of $\mathbb{H} P^{m-1}$ by an $(m-2)$-dimensional subtorus of a maximal torus $T^{m}$ in $\operatorname{Sp}(m)$ are selfdual Einstein metrics of positive scalar curvature with $T^{2}$ symmetry. These quotients were first studied by Galicki-Lawson [19] and Boyer-Galicki-Mann-Rees [7]; they are globally defined on compact orbifolds. Hence if we could obtain explicitly the relation between these quaternion-kähler quotients and Theorem 1.1, then we would have explicit formulae for the (hithertoo only implicit) Galicki-Lawson metrics and their generalizations.

Quaternion-kähler quotients of a quaternion-kähler manifold $Q$ may be related to hyperkähler quotients of its Swann bundle $\mathcal{U}(Q)$. Indeed the symmetry group lifts to an action on $\mathcal{U}(Q)$ preserving the hyperkähler structure, and commuting with the $\mathrm{CO}(3)$-action. The momentum map of this action is a $\mathrm{CO}(3)$ - and $G$-equivariant map $\mu: \mathcal{U}(Q) \rightarrow \operatorname{Im} \mathbb{H} \otimes \mathfrak{g}^{*}$, where $\operatorname{Im} \mathbb{H}$ carries the standard representation of $\mathrm{CO}(3)$ and $\mathfrak{g}$ is the Lie algebra of $G$, so that $\mathfrak{g}^{*}$ is the coadjoint representation. The hyperkähler quotient of $\mathcal{U}(Q)$, given by $\mathcal{U}(Q) / / / G=$ $\mu^{-1}(0) / G$, is therefore hyperkähler with a $\mathrm{CO}(3)$-action. (It may or may not be a manifold, but in any case the geometry of the local quotient is well-defined.) Swann proved that $\mathcal{U}(Q) / / / G$ is the Swann bundle of the quaternion-kähler quotient of $Q$ [36]. Indeed, when taking quotients of $\mathbb{H} P^{m-1}$ one often works in homogeneous coordinates, and this amounts to working on (the double cover of) the Swann bundle $\left(\mathbb{H}^{m} \backslash\{0\}\right) /\{ \pm 1\}$. 
Now hyperkähler quotients of $\mathbb{H}^{m}$ by tori are well understood. Let $T^{m}$ be the maximal torus of $\operatorname{Sp}(m)$ acting on $\mathbb{H}^{m}$ by $\left(q_{1}, \ldots q_{m}\right) \mapsto$ $\left(e^{i t_{1}} q_{1}, \ldots e^{i t_{m}} q_{m}\right)$. We can describe an $(m-2)$-subtorus of $T^{m}$ by declaring that its Lie algebra is the kernel of a map $\mathbb{R}^{m} \rightarrow \mathbb{R}^{2}$ sending the standard basis $e_{1}, \ldots e_{m}$ (the generators of the chosen $m$-torus) to some given $\alpha_{1}, \ldots \alpha_{m} \in \mathbb{R}^{2}$. Evidently $\alpha_{1}, \ldots \alpha_{m}$ must be rational (up to an overall factor) in order that the kernel of the map $\mathbb{R}^{m} \rightarrow \mathbb{R}^{2}$ is the Lie algebra of a subtorus. However, even without this condition, we can still consider the local hyperkähler quotient of $\mathbb{H}^{m}$ by an $(m-2)$ dimensional family of commuting triholomorphic Killing fields.

Specializing a result of Bielawski-Dancer [5] to the case of interest, we learn that the hyperkähler quotient of $\mathbb{H}^{m} \backslash\{0\}$ by this subtorus (with zero momentum map in order to obtain the Swann bundle of a quaternion-kähler quotient) is given by the generalized Gibbons-Hawking Ansatz with

$$
\Phi_{i j}=\sum_{k=1}^{m} \frac{\left(\alpha_{k}\right)_{i}\left(\alpha_{k}\right)_{j}}{r_{k}},
$$

where $r_{k}=\left|\left(\alpha_{k}\right)_{1} \boldsymbol{x}_{1}+\left(\alpha_{k}\right)_{2} \boldsymbol{x}_{2}\right|$.

Note that $\mathbb{R}^{2}$ here is the Lie algebra of the quotient torus. In our setting this is the vector space $\mathbb{W}$. Then $\alpha_{1}, \ldots \alpha_{m}$ define $m$ twistors on $\mathcal{H}^{2}$, which we write as $\varphi_{k}=\left[\begin{array}{l}a_{k} \\ b_{k}\end{array}\right]$, where $a_{k}=-\left(\alpha_{k}\right)_{2}, b_{k}=\left(\alpha_{k}\right)_{1}$. These $m$ twistors must determine the hyperbolic eigenfunction $F$ in some way. Since $\underline{\Phi}$ is $d \widetilde{F}$, we compute that

$$
\widetilde{F}=\sum_{k=1}^{m} r_{k}=\sum_{k=1}^{m}\left|b_{k} \boldsymbol{x}_{1}-a_{k} \boldsymbol{x}_{2}\right|
$$

and therefore

$$
F=\sum_{k=1}^{m} \frac{\sqrt{a_{k}^{2} \rho^{2}+\left(a_{k} \eta-b_{k}\right)^{2}}}{\sqrt{\rho}}=\sum_{k=1}^{m}\left|\varphi_{k}\right|,
$$

where $\left|\varphi_{k}\right|$ is the pointwise norm of the twistor $\left[\begin{array}{l}a_{k} \\ b_{k}\end{array}\right]$. Indeed, it is straightforward to check that the norm of a twistor $\varphi$ is a hyperbolic eigenfunction: without loss of generality we can take $\varphi=\left[\begin{array}{l}0 \\ 1\end{array}\right]=m_{0} / \sqrt{\rho}$, so that $F=1 / \sqrt{\rho}$.

For this 'monopole solution' $F$, the pencil of solutions of the Joyce equation degenerates: applying $F$ to $\left(-(a \eta-b) m_{0}+a \rho m_{1}\right) / \sqrt{\rho}$, using Proposition 7.1, gives $\Phi=2 a \mu_{1}$, which is the solution of the Joyce 
equation found by Joyce [26]. Joyce superposed this solution with its image under hyperbolic isometries in order to obtain his explicit metrics on $n \mathbb{C} P^{2}$.

The same trick yields interesting metrics here. For the monopole solution, $F=1 / \sqrt{\rho}, \frac{1}{4} F^{2}-\rho^{2}\left(F_{\rho}^{2}+F_{\eta}^{2}\right)$ is identically zero, and so we only obtain a selfdual Einstein metric only on the empty set! However for $m>1$ the ' $m$-pole' solutions $F=\sum_{k=1}^{m}\left|\varphi_{k}\right|$ yield quaternion-kähler quotients of $\mathbb{H} P^{m-1}$. Let us state our result more precisely.

Theorem 9.1. Let $\mathbb{R}^{m}$ be the Lie algebra of the maximal torus of $\operatorname{Sp}(m)$ which acts on $\mathbb{H} P^{m-1}$ by $\left[q_{1}: \ldots: q_{m}\right] \mapsto\left[e^{i t_{1}} q_{1}: \ldots: e^{i t_{m}} q_{m}\right]$. Let $M^{4}$ be the local quaternion-kähler quotient of $\mathbb{H} P^{m-1}$ by the $(m-2)$ dimensional family of Killing fields in the kernel of the map $\mathbb{R}^{m} \rightarrow \mathbb{W}$ sending the standard basis $e_{1}, \ldots e_{m}$ to $\varphi_{1}, \ldots \varphi_{m} \in \mathbb{W}$.

Then the selfdual Einstein metric on $M^{4}$ is given by (1.1) with $F=$ $\sum_{k=1}^{m}\left|\varphi_{k}\right|$.

The solutions corresponding to reductions of $\mathbb{H} P^{m-1}$ by an $(m-$ 2 )-torus yield the compact selfdual Einstein orbifolds we seek. These global reductions arise when $\varphi_{1}, \ldots \varphi_{m} \in \mathbb{W}$ span a 2-dimensional vector space over the rationals, i.e., when they can be chosen to have rational components.

The description of these metrics in terms of the hyperbolic plane links the geometry to the topological analysis of Boyer-Galicki-MannRees [7], who describe a $T^{2}$-invariant cell decomposition of their orbifolds over the closed disc, where the principal orbits fibre over the open disc and the special orbits fibre over the boundary. If we identify the open disc with the hyperbolic plane $\mathcal{H}^{2}$, then the boundary is the circle at infinity $P(\mathbb{W})$. The $m$ twistors determine $m$ marked points on this circle, corresponding to the fixed points of the action.

Non-compact analogues of these metrics may be obtained by taking quaternion-kähler quotients of quaternionic hyperboloids $\mathbb{H} \mathcal{H}^{p-1, q}$ and $\mathbb{H} \mathcal{H}^{p, q-1}$ by an $(m-2)$-subtorus of a Cartan subgroup of $\operatorname{Sp}(p, q), p+q=$ $m$. This includes, for instance, quotients of quaternionic hyperbolic space $\mathbb{H} \mathcal{H}^{m-1}$ when $\{p, q\}=\{m-1,1\}[17,18]$.

Such metrics may also be viewed as analytic continuations. Indeed, both the constructions of this paper and the hyperkähler and quaternion-kähler quotients may be carried out in the holomorphic category, and from this point of view real metrics are obtained by taking real slices of such complex metrics. In the holomorphic category, $|\varphi|$ must be taken to be a choice of branch of the square root of the com- 
plex bilinear pointwise inner product of $\varphi$ with itself. Other real slices are obtained by replacing a sum of real twistors either by a sum of complex conjugate twistors, or by a difference of real twistors, leading to multipole solutions of the form

$$
\begin{aligned}
F=\sum_{k=1}^{r}\left(\left|\varphi_{2 k-1}+\boldsymbol{i} \varphi_{2 k}\right|+\mid\right. & \left.\varphi_{2 k-1}-\boldsymbol{i} \varphi_{2 k} \mid\right) \\
& +\sum_{k=r+1}^{p}\left(\left|\varphi_{2 k-1}\right|-\left|\varphi_{2 k}\right|\right)+\sum_{k=2 p+1}^{p+q}\left|\varphi_{k}\right|,
\end{aligned}
$$

where $\varphi_{1}, \ldots \varphi_{p+q}$ are real and $0 \leqslant r \leqslant p$. (The norm of a complex twistor vanishes on the hyperbolic plane and so the complex conjugate pairs are only defined on a branched cover.)

The freedom here corresponds to the fact that $\operatorname{Sp}(p, q)$ does not have a unique Cartan subgroup up to conjugacy: for each $\operatorname{Sp}(1,1)$ factor, we can take either an $S^{1} \times \mathbb{R}$ subgroup or a $T^{2}$ subgroup, using (for instance) elements of the form

$$
\left(\begin{array}{ll}
e^{i t_{1}} \cosh t_{2} & e^{i t_{1}} \sinh t_{2} \\
e^{i t_{1}} \sinh t_{2} & e^{i t_{1}} \cosh t_{2}
\end{array}\right) \quad \text { or } \quad\left(\begin{array}{cc}
e^{i t_{1}} & 0 \\
0 & e^{i t_{2}}
\end{array}\right)
$$

in $\mathrm{U}(1,1)$. Hence if we suppose $p \leqslant q$ and write $\mathbb{H}^{p, q}=\left(\mathbb{H}^{1,1}\right)^{p} \times \mathbb{H}^{q-p}$, then we obtain a Cartan subgroup of $\operatorname{Sp}(1,1) \times \ldots \times \operatorname{Sp}(1,1) \times \operatorname{Sp}(q-p)$ of the form

$$
\left(S^{1} \times \mathbb{R}\right)^{r} \times T^{2(p-r)} \times T^{q-p} \leqslant \operatorname{Sp}(1,1)^{r} \times \operatorname{Sp}(1,1)^{p-r} \times \operatorname{Sp}(q-p) .
$$

We should remark, however, that in addition to Cartan subgroups, there are also maximal abelian subgroups containing nilpotent elements. We believe these yield a mild generalization of $m$-pole solutions, in which multipoles can become infinitesimal in a limiting process, such as the dipole $\lim _{\varepsilon \rightarrow 0} \frac{1}{\varepsilon}\left(\left|\varphi_{1}+\varepsilon \varphi_{2}\right|-\left|\varphi_{1}-\varepsilon \varphi_{2}\right|\right)$. We shall not study this kind of solution here.

The global behaviour of the $m$-pole solutions can be approached either via the quaternion-kähler quotient, as in $[7,17,18]$, or via compactification arguments based on local models, as discussed by Joyce [26]. A detailed analysis of this would take us to far afield here, so we turn instead to examples. 


\section{Examples}

In this section we study the simplest nontrivial examples of multipole selfdual Einstein metrics. An $m$-pole solution $F$ is determined, up to sign and reality choices, by $m$ distinct elements of the 2-dimensional vector space $\mathbb{W}$ of twistors, and conversely $F$ determines the twistors up to sign. Concretely, with respect to a choice of unimodular basis, we may write these elements as $\left[\begin{array}{l}a_{k} \\ b_{k}\end{array}\right]$. Now the group $\mathrm{SL}(\mathbb{W})$ acts naturally on the set of $m$-tuples of elements of $\mathbb{W}$, yielding equivalent solutions, so there is really only a $2 m-3$ parameter family of solutions. Furthermore, the solutions $F$ and $\lambda F$ yield the same Einstein metric for any $\lambda \neq 0$, so the moduli space actually has dimension $2 m-4$.

We first remark that the dipole solutions $(m=2)$ yield only hyperbolic and spherical metrics. More precisely, using the SL(W) freedom, we take the two twistors to be $\left[\begin{array}{l}a \\ 0\end{array}\right]$ and $\left[\begin{array}{l}0 \\ a\end{array}\right]$. Allowing for the homothety freedom, we are then left with three choices for the solution:

or

$$
\begin{aligned}
F^{+} & =\frac{1+\sqrt{\rho^{2}+\eta^{2}}}{\sqrt{\rho}}, \quad F^{-}=\frac{1-\sqrt{\rho^{2}+\eta^{2}}}{\sqrt{\rho}} \\
F^{c} & =\frac{\sqrt{\rho^{2}+(\eta+\boldsymbol{i})^{2}}+\sqrt{\rho^{2}+(\eta-\boldsymbol{i})^{2}}}{\sqrt{\rho}} .
\end{aligned}
$$

The first of these gives the spherical metric, while the other two give the hyperbolic metric with inequivalent torus actions.

We now consider the case $m=3$, when the moduli space is 2 dimensional. This case is particular interesting since selfdual Einstein metrics in this family have been studied in many places by diverse methods.

(i) In the context of finding selfdual Einstein metrics of negative scalar curvature with prescribed conformal infinity [27], the second author found complete examples on the 4-ball [31], depending on a single parameter in $(-1, \infty)$ (denoted $m^{2}$ there). It was later realized $[28,22]$ that when this parameter is $(2-n) / n$ (for $n \in \mathbb{Z}$, $n \geqslant 3$ ), analytic continuations of these metrics are complete on $\mathcal{O}(n) \rightarrow \mathbb{C} P^{1}$ (and are conformally related to LeBrun's scalar-flat Kähler metrics on $\mathcal{O}(-n))$.

(ii) By taking quaternion-kähler quotients of $\mathbb{H} P^{2}$ by $S^{1}$, Galicki and Lawson [19] found selfdual Einstein metrics of positive scalar curvature on certain compact orbifolds $\mathcal{O}_{q, p}(p, q$ coprime with $0<$ 
$q / p \leqslant 1)$. Negative scalar curvature analogues and generalizations have also been studied $[17,18]$.

(iii) A subfamily of the $m=3$ metrics have local cohomogeneity one, and are therefore bi-axial Bianchi metrics, which have been studied in many places, in particular [3]. The metrics in (i) are all in this subfamily. Furthermore, the quaternion-kähler quotients considered in detail in (ii) are mainly the local cohomogeneity one examples, although the general case is analogous [19, Remark $4.27]$.

(iv) Apostolov and Gauduchon [2] classify explicitly selfdual Einstein Hermitian metrics, i.e., admitting a selfdual complex structure: such metrics automatically have torus symmetry. They are conformal to selfdual Kähler metrics, which have been classified by Bryant [8] as the specialization to four dimensions of Kähler metrics with vanishing Bochner tensor. Conversely a generic selfdual Kähler metric is locally conformally Einstein. Apostolov and Gauduchon also show that quaternion-kähler quotients of $\mathbb{H} P^{2}$ and quaternionic hyperbolic space $\mathbb{H} \mathcal{H}^{2}$ by $S^{1}$ or $\mathbb{R}$ are selfdual Hermitian (and the same is true for $\mathbb{H} \mathcal{H}^{1,1}$ ). In particular the 3 -pole solutions are all Hermitian.

(v) These solutions have recently been studied by Casteill, Ivanov and Valent [12], using the harmonic superspace approach.

In view of all this work, we cannot claim that the $m=3$ examples are new. Nevertheless, in addition to presenting a unified treatment, we are able to give explicitly the parameter values yielding complete 3 -pole metrics on the 4-ball. At the end of the section, we shall use a perturbation argument to obtain complete $m$-pole metrics on the 4-ball for any $m$, showing that the moduli space is infinite dimensional.

Consider then the general 3-pole solution. Using the SL(WV) freedom we may write this in the form:

$$
F=\frac{a}{\sqrt{\rho}}+\frac{b+c / m}{2} \frac{\sqrt{\rho^{2}+(\eta+m)^{2}}}{\sqrt{\rho}}+\frac{b-c / m}{2} \frac{\sqrt{\rho^{2}+(\eta-m)^{2}}}{\sqrt{\rho}}
$$

where $|m|=1$, but $m$ can be imaginary or real, $-m^{2}= \pm 1$. We refer to these as Type I and Type II solutions respectively, after the 
Eguchi-Hanson I and II metrics. It is convenient to work in (EguchiHanson)-like coordinates

$$
\rho=\sqrt{R^{2} \pm 1} \cos \theta, \quad \eta=R \sin \theta
$$

with $\theta \in(-\pi / 2, \pi / 2)$ so that

$$
\sqrt{\rho} F=a+b R+c \sin \theta
$$

and

$$
\rho^{-1}\left(\frac{1}{4} F^{2}-\rho^{2}\left(F_{\rho}^{2}+F_{\eta}^{2}\right)\right)=\frac{b(a R \mp b)+c(a \sin \theta+c)}{R^{2} \pm \sin ^{2} \theta} .
$$

Note that the zero-set of $F$ is a conformal infinity of the selfdual Einstein metric, which has negative scalar curvature there. On the other hand the zero-set of $\frac{1}{4} F^{2}-\rho^{2}\left(F_{\rho}^{2}+F_{\eta}^{2}\right)$ is a singularity separating domains of positive and negative scalar curvature, and the metric is incomplete there. Let us consider the Type I and Type II metrics separately.

In the Type I case, $F$ is only globally defined on a branched double cover of the hyperbolic plane: we can regard $R=0$ as the branch cut between $R \geqslant 0$ and $R \leqslant 0$. Without loss of generality, we can assume $a$ is nonzero and use the homothety freedom to set $a=1$. We can also suppose $b, c \geqslant 0$ (using $R \mapsto-R$ and $\theta \mapsto-\theta$ ).

When $b$ is nonzero we have, for each $\theta \in(-\pi / 2, \pi / 2)$, a unique value of $R$, namely $R_{\infty}=-(1+c \sin \theta) / b$, at which $F=0$, and a unique value $R_{ \pm}=\left(b^{2}+c^{2}+c \sin \theta\right) / b$ where $\frac{1}{4} F^{2}-\rho^{2}\left(F_{\rho}^{2}+F_{\eta}^{2}\right)=0$. When $c=0$, $R_{\infty}=-1 / b$ and $R_{ \pm}=b$ : this is the case that the selfdual Einstein metric is a bi-axial Bianchi IX metric, i.e., of local cohomogeneity one under $\mathrm{U}(2)$, and the distinguished Einstein-Weyl quotient (by the centre of $\mathrm{U}(2))$ is $S^{3}$ [31].

In general, one checks that $R_{\infty}<R_{ \pm}$for all $\theta$. Hence there are three domains of definition:

- $R \in\left(-\infty, R_{\infty}\right)$ : here the metric has negative scalar curvature and yields a complete metric on the ball $B^{4}$ with a conformal infinity at $R=R_{\infty}$.

- $R \in\left(R_{\infty}, R_{ \pm}\right)$: the metric still has negative scalar curvature, but has an unremovable singularity at $R=R_{ \pm}$.

- $R \in\left(R_{ \pm}, \infty\right)$ : the metric now has positive scalar curvature, again with an unremovable singularity at $R=R_{ \pm}$. 
The complete domain was found by Galicki [18], although it is only more recently that it has been noticed that these metrics are more general than those of [31].

If $b=0$ then there are two nontrivial cases: for $c>1$, there is a conformal infinity at $\sin \theta=-1 / c$ and no singularity, while for $c<1$ there is a singularity at $\sin \theta=-c$ and no conformal infinity. These turn out to be Bianchi VIII metrics, i.e., have local cohomogeneity one under $\operatorname{GL}(2, \mathbb{R})$, and the distinguished Einstein-Weyl quotient is $\mathcal{H}^{3}$ [11]. For $c=1$ the metric is the Bergman metric on $\mathbb{C} \mathcal{H}^{2}$. We illustrate this discussion with a diagram (Figure 2) of the $(b, c)$-plane next to which we give a heuristic picture of the behaviour of $F$ on the branched double cover of the hyperbolic plane by shading the domain over which the Einstein metric has positive scalar curvature and indicating the conformal infinity in the domain of negative scalar curvature.

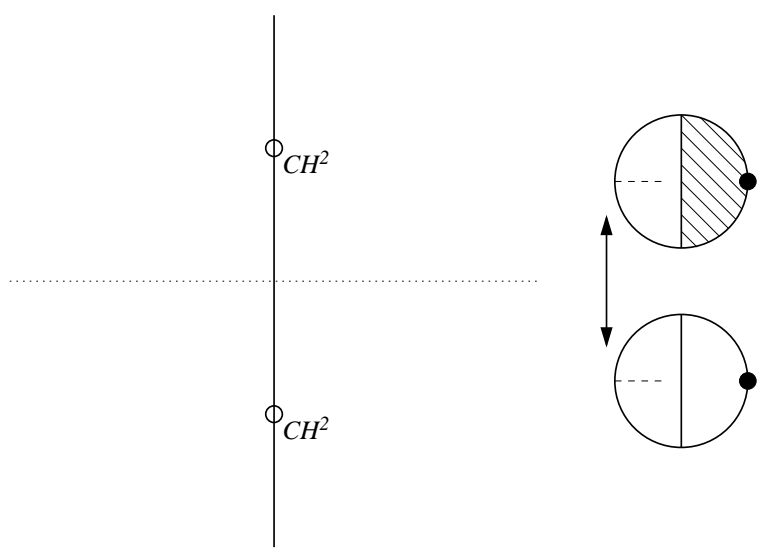

Figure 2.

For the Type II metrics the range of $R$ is $(1, \infty)$ and $F$ is globally defined on the hyperbolic plane. However the moduli space has a much richer structure and the analysis is more involved. The homothety freedom means that the Einstein metrics are parameterized by a point $[a, b+c, b-c]$ in $\mathbb{R} P^{2} \backslash\{[1,0,0],[0,1,0],[0,0,1]\}$. The lines joining the points $[1,0,0],[0,1,0]$ and $[0,0,1]$ represent dipole solutions, so the true moduli space is obtained by removing these lines and taking the quotient by the permutation group $\mathrm{Sym}_{3}$ of the coordinates. For convenience we shall only remove the line $a=0$, so that we can set $a=1$ and use inhomogeneous coordinates $(b, c)$ as in the Type I case. On the lines $b= \pm c$ the selfdual Einstein metric is the hyperbolic metric for 
$b<0$ and the spherical metric for $b>0$.

First note that $(b, c)=(1,0)$ (the fixed point $[1,1,1]$ ) gives the Fubini-Study metric on $\mathbb{C} P^{2}$, whereas the points $(0,1),(0,-1)$ and $(-1,0)$ yield the Bergman metric on $\mathbb{C H}^{2}$ (cf. [19,31]). Along the lines joining these four points, we have bi-axial Bianchi metrics with distinguished quotient $\mathcal{H}^{3}$ : along the lines joining $(1,0)$ to the others, the metric is Bianchi IX, whereas on the lines between $(0,1),(0,-1)$ and $(-1,0)$, the metric is Bianchi VIII.

As in the Type I case, the zero-sets of $F$ and $\frac{1}{4} F^{2}-\rho^{2}\left(F_{\rho}^{2}+F_{\eta}^{2}\right)$ do not meet. This is a matter of checking that there are no simultaneous solutions of

$$
\begin{aligned}
b R+c S+1 & =0 \\
b R+b^{2}-c S-c^{2} & =0
\end{aligned}
$$

with $S=\sin \theta \in(-1,1), R>1$. The solution of (10.1) for $b, c \neq 0$ is $R=-\left(1+b^{2}-c^{2}\right) / 2 b, S=-\left(1-b^{2}+c^{2}\right) / 2 c$ which satisfies $b^{2}\left(R^{2}-1\right)=$ $c^{2}\left(S^{2}-1\right)$. Hence we see that we cannot have $S \in[-1,1], R \in[1, \infty]$ unless $(b, c)$ lies on one of the Bianchi VIII lines $b=0, b+c+1=0$ or $b-c+1=0$, in which case there are solutions $R=\infty,(R, S)=(1,-1)$ and $(R, S)=(1,1)$ respectively, i.e., at the three marked points at infinity.

Analysing the equations in (10.1) separately, we can determine for which $(b, c)$ the selfdual Einstein metric has positive and/or negative scalar curvature domains, and whether there is a conformal infinity. The Bianchi VIII lines $b=0, b \pm c+1=0$ and the dipole lines $b= \pm c$ divide the $(b, c)$ plane into $1+3+3+6=13$ regions of four different types leading to the following picture of the (pre-)moduli space (Figure 3 ):

In this figure the Bianchi VIII lines are solid, the Bianchi IX lines are dotted (and do not bound regions), while the dipole lines are dashed. We label the type of the region by $A, B, C, D$ and sketch the topology of the zero sets in the hyperbolic disc as before. In these regions of the moduli space the geometry of the selfdual Einstein metric is as follows:

(A) The metric has positive scalar curvature and no singularities for $R \in(1, \infty)$. When $(b, c)=(1,0)$ it is the Fubini-Study metric, while for other rational values the metric may be compactified on a weighted projective space $\mathbb{C} P^{[p, q, r]}$, and the selfdual Einstein metrics are Hermitian $[2,8,19]$. 


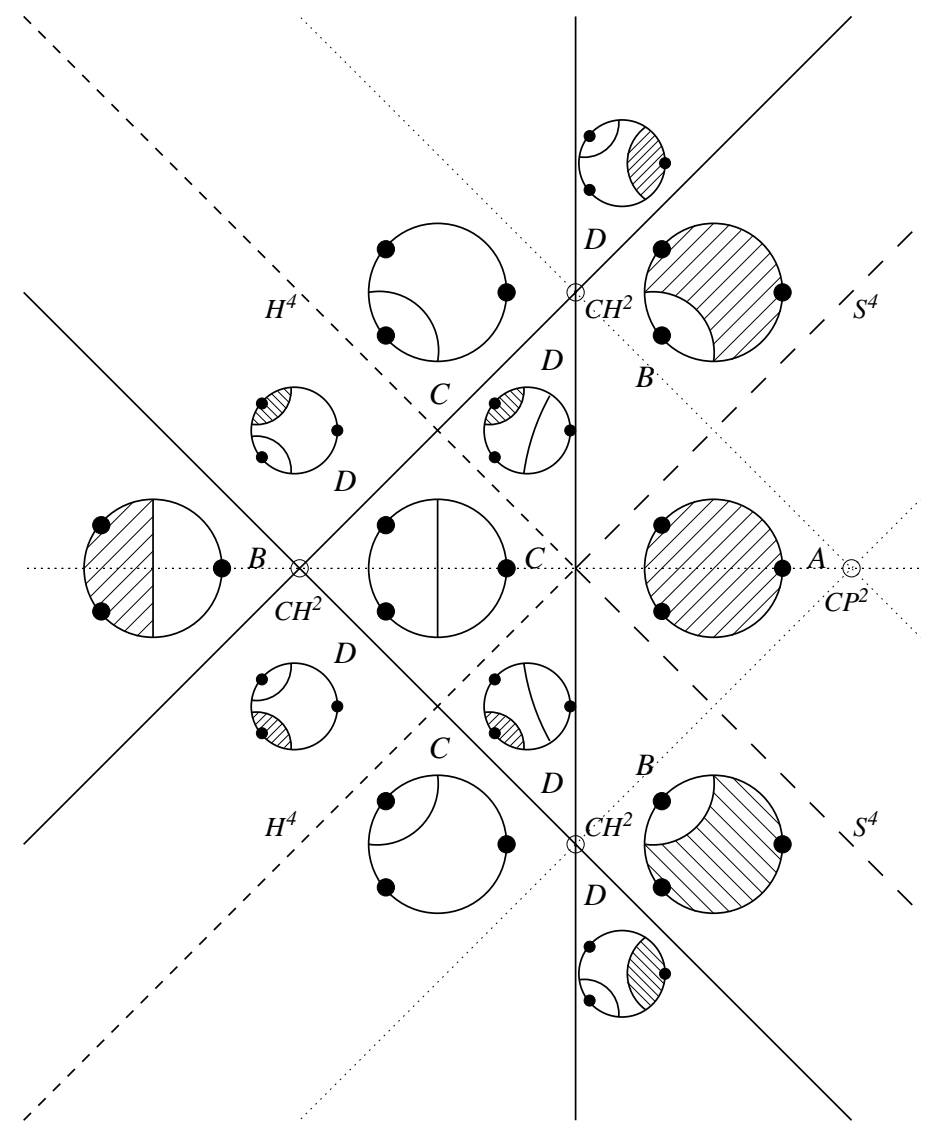

Figure 3.

(B) The metric has a positive and a negative scalar curvature domain separated by an unremovable singularity.

(C) The metric has two domains of negative scalar curvature separated by a conformal infinity. On one side of the conformal infinity the metric is complete on $B^{4}$. For rational parameter values, the metric on the other side yields a complete metric on $\mathcal{O}(n) \rightarrow \mathbb{C} P^{1}$ or an orbifold generalization of this $[17,22,28,31]$.

(D) The metric has two domains of negative scalar curvature and one of positive scalar curvature. On one side of the conformal infinity we obtain a complete metric on $B^{4}$. This is similar to the behaviour in the Type I case.

It is also fairly clear from Figure 3 what happens as $(b, c)$ passes 
from one region to another. Along the dipole lines $b= \pm c$, a bubble of positive or negative scalar curvature appears or disappears at one of the marked points, whereas along the Bianchi VIII lines, either the singularity or the conformal infinity passes through one of the marked points, creating or destroying a domain of positive or negative scalar curvature as it does.

The $R, S$ coordinates give a reasonably simple formula for the metric.

$$
\begin{aligned}
g= & \frac{b^{2}-c^{2}+a(b R-c S)}{(a+b R+c S)^{2}}\left(\frac{d R^{2}}{R^{2}-1}+\frac{d S^{2}}{1-S^{2}}\right) \\
+ & \frac{1}{(a+b R+c S)^{2}\left(b^{2}-c^{2}+a(b R-c S)\right)\left(R^{2}-S^{2}\right)} \\
& \cdot\left(\left(R^{2}-1\right)\left(1-S^{2}\right)((b R-c S) d \phi+(c R-b S) d \psi)^{2}\right. \\
& \quad+\left(\left(b\left(R^{2}-1\right) S+c\left(1-S^{2}\right) R\right) d \phi\right. \\
& \left.\left.\quad+\left(c\left(R^{2}-1\right) S+b\left(1-S^{2}\right) R+a\left(R^{2}-S^{2}\right)\right) d \psi\right)^{2}\right) .
\end{aligned}
$$

In particular, the metric is rational. This is not surprising in view of the work of Apostolov-Gauduchon [2] and Bryant [8]: the 3-pole metrics are all Hermitian, and are given explicitly in terms of a fourth order polynomial $P(y)$ whose roots sum to zero. Despite the superficial resemblence of this formula to [8, Section 4.3.2] (see also [1]), the precise relationship is rather complicated. By computing the selfdual Weyl curvature $W^{+}$of the Einstein metric $g$, we find that the selfdual Kähler metric $\left|W^{+}\right|^{2 / 3} g$ - see $[2,14]$ - is (a constant multiple of) $(a+b R+$ $c S)^{2} g /\left(b^{2}-c^{2}+a(b R-c S)\right)^{2}$ with Kähler form

$$
\begin{aligned}
& \frac{1}{\left(b^{2}-c^{2}+a(b R-c S)\right)^{2}} \\
& \cdot((d \phi \wedge(b d R-c d S)+d \psi \wedge((c+a S) d R-(b+a R) d S))) .
\end{aligned}
$$

The momentum maps of $\partial_{\phi}$ and $\partial_{\psi}$ are therefore (up to an affine transformation)

$$
\frac{b R-c S}{b^{2}-c^{2}+a(b R-c S)} \quad \text { and } \quad \frac{c R-b S}{b^{2}-c^{2}+a(b R-c S)} .
$$

According to [8], these must be affine linear combinations of the trace $u_{1}=y_{1}+y_{2}$ and the pfaffian $u_{2}=y_{1} y_{2}$ of the normalized Ricci form of the Kähler metric, and by $[2,14] u_{1}^{2}$ is the conformal factor from the 
Einstein metric to the Kähler metric. For generic $a, b, c$, this allows us to put the Kähler metric into the form of $[8,1]$ :

$$
\begin{aligned}
& \left(y_{1}-y_{2}\right)\left(\frac{d y_{1}^{2}}{P\left(y_{1}\right)}-\frac{d y_{2}^{2}}{P\left(y_{2}\right)}\right) \\
& \quad+\frac{1}{y_{1}-y_{2}}\left(P\left(y_{1}\right)\left(d t_{1}+y_{2} d t_{2}\right)^{2}-P\left(y_{2}\right)\left(d t_{1}+y_{1} d t_{2}\right)^{2}\right)
\end{aligned}
$$

where $P(y)=\left(y-2 a b-b^{2}+c^{2}\right)\left(y+2 a b-b^{2}+c^{2}\right)\left(y-2 a c+b^{2}-c^{2}\right)(y+$ $\left.2 a c+b^{2}-c^{2}\right)$. We interpret this formula abstractly by noting that three twistors $\varphi_{1}, \varphi_{2}, \varphi_{3}$ give rise to three $\mathrm{SL}(\mathbb{W})$-invariants, namely the determinants $z_{1}=\varepsilon\left(\varphi_{2}, \varphi_{3}\right), z_{2}=\varepsilon\left(\varphi_{3}, \varphi_{1}\right)$ and $z_{3}=\varepsilon\left(\varphi_{1}, \varphi_{2}\right)$. Up to an overall sign, the roots of the polynomial $P(y)$ are then

$$
\begin{array}{ll}
r_{0}=\frac{1}{2}\left(z_{1}+z_{2}+z_{3}\right), & r_{1}=\frac{1}{2}\left(z_{1}-z_{2}-z_{3}\right), \\
r_{2}=\frac{1}{2}\left(-z_{1}+z_{2}-z_{3}\right), & r_{3}=\frac{1}{2}\left(-z_{1}-z_{2}+z_{3}\right) .
\end{array}
$$

Hence we have related the generic Type II 3-pole Einstein metrics to the Case 4 Kähler metrics of [8]. In a similar way, the generic Type I 3-pole Einstein metrics are related to Bryant's Case 1 Kähler metrics. The extra lines in our 3-pole moduli space are cohomogeneity one metrics, which are treated separately in $[2,8]$ because the trace and the pfaffian of the normalized Ricci form are not independent. On the other hand Bryant's Case 2 and Case 3 metrics are not covered by the 3-pole metrics, because $P(y)$ then has repeated roots. These correspond to quaternion-kähler quotients of $\mathbb{H} \mathcal{H}^{2}$ and $\mathbb{H} \mathcal{H}^{1,1}$ by a non-semisimple $S^{1}$ action. We could obtain them from a limiting process in which a dipole becomes infinitesimal.

Let us end by remarking that it is straightforward to obtain many complete selfdual Einstein metrics with $T^{2}$ symmetry on $B^{4}$. Such metrics arise when there is a domain of negative scalar curvature surrounding a single marked point and bounded by a conformal infinity. Starting with a known example we can deform $F$ slightly by adding additional monopole solutions at points on the other side of the conformal infinity. The zero-set of $F$ deforms smoothly and so the metric stays complete until the conformal infinity hits a fixed point. This argument yields not only the 3 -pole solutions, but $m$-pole solutions for any $m>2$. Hence the moduli space of smooth and complete torus-symmetric selfdual Einstein metrics on the ball is infinite dimensional, cf. [30].

We can also obtain infinite dimensional families of smooth complete metrics with other topologies, but we postpone the discussion of these examples to another occasion. 


\section{References}

[1] V. Apostolov, D.M.J. Calderbank \& P. Gauduchon, The geometry of weakly selfdual Kähler surfaces, Compositio Math., to appear, math.DG/0104233.

[2] V. Apostolov \& P. Gauduchon, Selfdual Einstein Hermitian four manifolds, Ann. Scuola Norm. Pisa, to appear, math.DG/0003162.

[3] L. Bérard-Bergery, Sur de nouvelles variétés riemanniennes d'Einstein, in 'Institut Élie Cartan', Univ. Nancy, Nancy, 1982, MR 85b:53048, Zbl 0544.53038.

[4] A.L. Besse, Einstein Manifolds, Ergeb. Math. Grenzgeb., 10, Springer, Berlin, 1987, MR 88f:53087, Zbl 0613.53001.

[5] R. Bielawski \& A.S. Dancer, The geometry and topology of toric hyperkähler manifolds, Comm. Anal. Geom. 8 (2000) 727-760, MR 2002c:53078.

[6] C.P. Boyer \& J.D. Finley, Killing vectors in self-dual Euclidean Einstein spaces, J. Math. Phys. 23 (1982) 1126-1130, MR 84f:53064.

[7] C.P. Boyer, K. Galicki, B.M. Mann \& E.G. Rees, Compact 3-Sasakian 7-manifolds with arbitrary second Betti number, Invent. Math. 131 (1998) 321-344, MR 99b:53066, Zbl 0901.53033.

[8] R.L. Bryant, Bochner-Kähler metrics, J. Amer. Math. Soc. 14 (2001) 623-715, MR 2002i:53096.

[9] D.M.J. Calderbank, The geometry of the Toda equation, J. Geom. Phys. 36 (2000) 152-162, MR 2001m:53083, Zbl 0979.53046.

[10] D.M.J. Calderbank \& H. Pedersen, Einstein-Weyl geometry, in 'Essays on Einstein Manifolds' (C. R. LeBrun \& M. Wang, eds.), Surveys in Differential Geometry VI, International Press, Cambridge, 1999, 387-423, MR 2002b:53062.

[11] D.M.J. Calderbank \& H. Pedersen, Selfdual spaces with complex structures, Einstein-Weyl geometry and geodesics, Ann. Inst. Fourier 50 (2000) 921-963, MR 2001h:53058, Zbl 0970.53027.

[12] P.Y. Casteill, E. Ivanov \& G. Valent, Quaternionic extension of the double TaubNUT metric, Phys. Lett. B 508 (2001) 354-364, CMP 1843 926, Zbl 0977.83070.

[13] B. de Wit, M. Roček \& S. Vandoren, Hypermultiplets, hyperkähler cones and quaternion-Kähler geometry, J. High Energy Phys. 01-02 (2001) Paper 39, MR 2002e:81217.

[14] A. Derdziński, Self-dual Kähler manifolds and Einstein manifolds of dimension four, Compositio Math. 49 (1983) 405-433, MR 84h:53060, Zbl 0527.53030.

[15] J.D. Finley \& M.V. Saveliev, Heavenly equation with one Killing vector and a cosmological term, Phys. Lett. A 162 (1992) 1-4, MR 92k:83025. 
[16] K. Galicki, A generalization of the momentum mapping construction for quaternionic Kähler manifolds, Comm. Math. Phys. 108 (1987) 117-138, MR 88f:53088, Zbl 0608.53058.

[17] K. Galicki, New matter couplings in $N=2$ supergravity, Nuclear Phys. B 289 (1987) 573-588, MR 88j:53076.

[18] K. Galicki, Multi-centre metrics with negative cosmological constant, Class. Quantum Grav. 8 (1991) 1529-1543, MR 92i:53040, Zbl 0737.53061.

[19] K. Galicki \& H.B. Lawson, Jr., Quaternionic reduction and quaternionic orbifolds, Math. Ann. 282 (1988) 1-21, MR 89m:53075, Zbl 0628.53060.

[20] P. Gauduchon, Surfaces kähleriennes dont la courbure vérifie certaines conditions de positivité, in 'Géométrie Riemannienne en Dimension 4' (Séminaire Besse 1978/1979, L. Bérard-Bergery, M. Berger \& C. Houzel, eds.), Textes Math., 3, CEDIC, Paris, 1981, 220-263, MR 86i:53020, Zbl 0513.53058.

[21] G.W. Gibbons \& S.W. Hawking, Gravitational multi-instantons, Phys. Lett. B 78 (1978) 430-432.

[22] N.J. Hitchin, Twistor spaces, Einstein metrics and isomonodromic deformations, J. Differential Geom. 42 (1995) 30-112, MR 96g:53057, Zbl 0861.53049.

[23] N.J. Hitchin, A. Karlhede, U. Lindström \& M. Roček, Hyper-Kähler metrics and supersymmetry, Comm. Math. Phys. 108 (1987) 535-589, MR 88g:53048, Zbl 0612.53043.

[24] B. Iversen, Hyperbolic Geometry, Cambridge University Press, Cambridge, 1992, MR 94b:51023, Zbl 0766.51002.

[25] P.E. Jones \& K.P. Tod, Minitwistor spaces and Einstein-Weyl spaces, Class. Quantum Grav. 2 (1985) 565-577, MR 87b:53115, Zbl 0575.53042.

[26] D.D. Joyce, Explicit construction of self-dual 4-manifolds, Duke Math. J. 77 (1995) 519-552, MR 96d:53049, Zbl 0855.57028.

[27] C.R. LeBrun, $\mathcal{H}$-space with a cosmological constant, Proc. Roy. Soc. London A 380 (1982) 171-185, MR 83d:83019, Zbl 0549.53042.

[28] C.R. LeBrun, Counterexamples to the generalized positive action conjecture, Comm. Math. Phys. 118 (1988) 591-596, MR 89f:53107, Zbl 0659.53050.

[29] C.R. LeBrun, Explicit self-dual metrics on $\mathbb{C} P^{2} \# \ldots \# \mathbb{C} P^{2}$, J. Differential Geom. 34 (1991) 223-253, MR 92g:53040, Zbl 0725.53067.

[30] C.R. LeBrun, On complete quaternionic-Kähler manifolds, Duke Math. J. 63 (1991) 723-743, MR 92i:53042, Zbl 0764.53045.

[31] H. Pedersen, Einstein metrics, spinning top motions and monopoles, Math. Ann. 274 (1986) 35-39, MR 87i:53070, Zbl 0566.53058. 
[32] H. Pedersen \& Y.S. Poon, Hyper-Kähler metrics and a generalization of the Bogomolny equations, Comm. Math. Phys. 117 (1988) 569-580, MR 89f:53069.

[33] M. Pontecorvo, On twistor spaces of anti-self-dual hermitian surfaces, Trans. Amer. Math. Soc. 331 (1992) 653-661, MR 92h:32047, Zbl 0754.53053.

[34] M. Przanowski, Killing vector fields in self-dual, Euclidean Einstein spaces with $\lambda \neq 0$, J. Math. Phys. 32 (1991) 1004-1010, MR 91m:83029, Zbl 0737.53073.

[35] S.M. Salamon, Quaternionic Kähler manifolds, Invent. Math. 67 (1982) 143-171, MR 83k:53054, Zbl 0486.53048.

[36] A. Swann, Hyper-Kähler and quaternionic Kähler geometry, Math. Ann. 289 (1991) 421-450, MR 92c:53030, Zbl 0718.53051.

[37] K.P. Tod, Cohomogeneity-one metrics with self-dual Weyl tensor, in 'Twistor Theory' (Plymouth, 1993, S.A. Huggett, ed.), Lecture Notes in Pure and Appl. Math., 169, Marcel Dekker, New York, 1995, 171-184, MR 95i:53056, Zbl 0827.53017.

[38] K.P. Tod, Scalar-flat Kähler and hyper-Kähler metrics from Painlevé-III, Class. Quantum Grav. 12 (1995) 1535-1547, MR 96f:53068, Zbl 0828.53061.

[39] K.P. Tod, The SU( $\infty)$ Toda field equation and special four-dimensional metrics, in 'Geometry and Physics' (Aarhus, 1995, J.E. Andersen, J. Dupont, H. Pedersen \& A. Swann, eds.), Lecture Notes in Pure and Appl. Math., 184, Marcel Dekker, New York, 1997, 307-312, MR 98a:53068, Zbl 0876.53026.

[40] R.S. Ward, Einstein-Weyl spaces and SU( $\infty)$ Toda fields, Class. Quantum Grav. 7 (1990) L95-L98, MR 91g:83019, Zbl 0687.53044.

\author{
UNIVERSITY OF EDINBURGH \\ EDINBURGH EH9 3JZ \\ SCOTLAND \\ SDU-ODENSE UNIVERSITY \\ DK-5230 Odense M \\ DENMARK
}

